\title{
Workbook for Statistics
}

\section{Petra Schreiberová, Marcela Rabasová}




\section{Introduction}

This material should provide a guide for the study of the subject Statistics at VSB - Technical University of Ostrava, either for Erasmus students or for students of the study programs taught in English.

\section{Thanks}

The text was written with the financial support of the project Technology for the Future 2.0, CZ.02.2.69/0.0/0.0/18_058/0010212. 


\section{Contents}

1 Combinatorics $\quad 5$

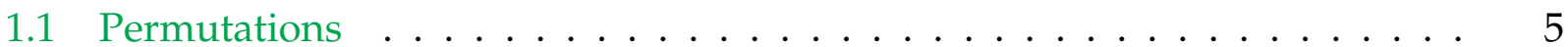

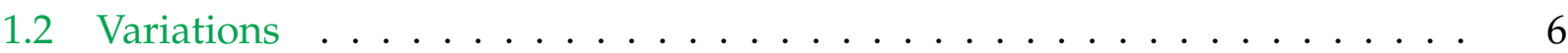

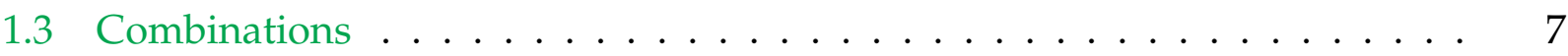

2 Basic probability 9

2.1 Random trial, random event . . . . . . . . . . . . . . . . . 9 9

2.2 Probability of random event . . . . . . . . . . . . . . . . . 11

2.3 Conditional probability, independent events . . . . . . . . . . . . . 12

2.4 The total probability theorem, Bayes' theorem . . . . . . . . . . . . . 14

3 Random variable $\quad 16$

3.1 Distribution function . . . . . . . . . . . . . . . . . 16

3.2 Probability function . . . . . . . . . . . . . . . . . . . 17

3.3 Probability density function . . . . . . . . . . . . . . . . . 19

3.4 Numerical characteristics . . . . . . . . . . . . . . . . . . 20

4 Discrete probability distribution $\quad 25$

5 Continuous probability distribution $\quad 29$

5.1 Normal approximation . . . . . . . . . . . . . . . 33

6 Descriptive statistics: summary numbers 35

6.1 Measures of location . . . . . . . . . . . . . . . 35

6.2 Measures of variability . . . . . . . . . . . . . . . 36

7 Grouped frequencies and graphical descriptions $\quad 39$

7.1 Stem-and-leaf display . . . . . . . . . . . . . . . . . . . . 39

7.2 Box plot (box-and-whisker plot) . . . . . . . . . . . . . . . . . . . . . . . . . . . . . . . . .

7.3 Bar chart (bar graph) . . . . . . . . . . . . . . . . . . . . 40

7.4 Graphs of continuous data . . . . . . . . . . . . . . . . . . 40

7.5 Histogram . . . . . . . . . . . . . . . . . . . . 41

7.6 Cumulative frequency diagram . . . . . . . . . . . . . . . 42

8 Sampling and combination of variables 43

8.1 Linear combination of independent variables . . . . . . . . . . . . . . . 43

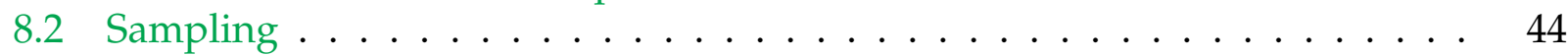

8.3 Central limit theorem . . . . . . . . . . . . . . . . . 45

9 Statistical inferences for the mean $\quad 47$

9.1 Hypothesis testing . . . . . . . . . . . . . . . . . . 47

9.2 Inferences for the mean $-H_{0}: \mu=\mu_{0} \ldots \ldots \ldots$

9.3 Confidence interval for the mean . . . . . . . . . . . . . . . 51

9.4 Comparison of sample means $-H_{0}: \mu_{1}=\mu_{2} \ldots \ldots \ldots$. . . . . . 52 
10 Regression and correlation analysis

10.1 Method of least squares . . . . . . . . . . . . . . . . . 56

10.2 Regression model validation . . . . . . . . . . . . . . . . . . . 58

10.3 Inferences for coefficients . . . . . . . . . . . . . . . . . . 60

10.4 Correlation . . . . . . . . . . . . . . . . . . . 62 


\section{Combinatorics}

\subsection{Permutations}

\section{Definition}

Permutations are arrangements of objects (with or without repetition) where the internal order is significant. The number of permutations of $n$ objects is the number of all different arrangements in which $n$ items can be placed.

\section{Formulas:}

- number of permutations of $n$ objects without repetition

$$
P(n)=n !
$$

- number of permutations of $n$ objects with repetitions

$$
P(n)^{*}=\frac{n !}{n_{1} ! n_{2} ! \cdots n_{k} !}
$$

- number of circular permutations of $n$ objects

$$
P(n)^{\circ}=(n-1) \text { ! }
$$

Remark

Excel - czech version:

$P(n)=\operatorname{PERMUTACE}(n ; n)$

$n !=$ FAKTORIÁL $(n)$

Excel - english version:

$P(n)=\operatorname{PERMUT}(n ; n)$

$n !=\mathrm{FACT}(n)$

Example 1

a) In how many ways can a group of eight persons arrange themselves in a row?

b) In how many ways can a group of eight persons arrange themselves in a circle?

c) How many different numbers can be created by using all the digits of the number 11212251 ?

a) $P(8)=8 !=8 \cdot 7 \cdot 6 \cdot 5 \cdot 4 \cdot 3 \cdot 2 \cdot 1=40320$

Excel: $P(8)=$ PERMUTACE $(8 ; 8)=40320$

b) $P(8)^{\circ}=(8-1) !=7 !=7 \cdot 6 \cdot 5 \cdot 4 \cdot 3 \cdot 2 \cdot 1=5040$

Excel: $P(7)=$ PERMUTACE $(7 ; 7)=5040$

c) $P(8)^{*}=\frac{8 !}{4 ! \cdot 3 ! \cdot 1 !}=280$

Excel: $P(8)^{*}=$ FAKTORIÁL $(8) /($ FAKTORIÁL $(4) *$ FAKTORIÁL $(3))=280$ 


\subsection{Variations}

\section{Definition}

Variations are arrangements of selections of objects (with or without repetition) where the internal order is significant. The number of $k$-element variations of $n$ objects is the number of all different arrangements of all different $k$-item selections from a group of $n$ items.

\section{Formulas:}

- number of $k$-element variations of $n$ objects without repetition

$$
V_{k}(n)=\frac{n !}{(n-k) !}
$$

- number of $k$-element variations of $n$ objects with repetitions

$$
V_{k}^{*}(n)=n^{k}
$$

\section{Remark}

Excel - czech version:

$V_{k}(n)=\operatorname{PERMUTACE}(n ; k)$

$V_{k}^{*}(n)=\operatorname{POWER}(n ; k)$

Excel - english version:

$P(n)=\operatorname{PERMUT}(n ; n)$

$n !=\operatorname{POWER}(n)$

\section{Example 2}

a) Specify the number of all possible three-digit codes in which the numbers can not be repeated.

b) Specify the number of all possible three-digit codes in which the numbers can be repeated.

c) In how many ways can be occupied medal positions by ten sprinters?

a) $V_{3}(10)=\frac{10 !}{(10-3) !}=\frac{10 !}{7 !}=\frac{10 \cdot 9 \cdot 8 \cdot 7 !}{7 !}=720$

Excel: $V_{3}(10)=\operatorname{PERMUTACE}(10 ; 3)=720$

b) $V_{3}^{*}(10)=10^{3}=1000$

Excel: $V_{3}^{*}(10)=\operatorname{POWER}(10 ; 3)=1000$

c) $V_{3}(10)=\frac{10 !}{(10-3) !}=720$

Excel: $V_{3}(10)=\operatorname{PERMUTACE}(10 ; 3)=720$ 


\subsection{Combinations}

\section{Definition}

Combinations are selections of objects (with or without repetition) where the internal order is not significant. The number of $k$-element combinations of $n$ objects is the number of all ways of choosing $k$ items from a group of $n$ items where we do not take the order into account.

\section{Formulas:}

- number of $k$-element combinations of $n$ objects without repetition

$$
C_{k}(n)=\left(\begin{array}{l}
n \\
k
\end{array}\right)=\frac{n !}{(n-k) ! \cdot k !}
$$

- number of $k$-element combinations of $n$ objects with repetition

$$
C_{k}^{*}(n)=\left(\begin{array}{c}
n+k-1 \\
k
\end{array}\right)
$$

\section{Remark}

Excel - czech version:

$C_{k}(n)=\operatorname{KOMBINACE}(n ; k)$

$C_{k}^{*}(n)=\operatorname{KOMBINACE}(n+k-1 ; k)$

Excel - english version:

$C_{k}(n)=\operatorname{COMBIN}(n ; k)$

$C_{k}^{*}(n)=\operatorname{COMBIN}(n+k-1 ; k)$

Example 3

a) Ten kinds of cakes are sold in the cake shop. How many options do we have to order eight cakes?

b) Ten kinds of cakes are sold in the cake shop. How many options do we have to order eight different cakes?

c) There are ten people waiting for a lift. How many options do we have to choose eight of them to go there?

a) $C_{8}^{*}(10)=\left(\begin{array}{c}10+8-1 \\ 8\end{array}\right)=\left(\begin{array}{c}17 \\ 8\end{array}\right)=\frac{17 !}{(17-8) ! \cdot 8 !}=24310$

Excel: $C_{8}^{*}(10)=\operatorname{KOMBINACE}(10+8-1 ; 8)=24310$

b) $C_{8}(10)=\left(\begin{array}{c}10 \\ 8\end{array}\right)=\frac{10 !}{(10-8) ! \cdot 8 !}=45$

Excel: $C_{8}(10)=\operatorname{KOMBINACE}(10 ; 8)=45$ 
c) $C_{8}(10)=\left(\begin{array}{c}10 \\ 8\end{array}\right)=45$

Excel: $C_{8}(10)=\operatorname{KOMBINACE}(10 ; 8)=45$

\section{Exercise 4}

a) How many options do we have to seat 8 students to 8 different computers?

b) How many different anagrams (words) can be created using all the letters of the word STATISTICS?

c) How many games will be played in an eight-team hockey tournament in which every team will play with every other team just once?

d) Ten friends have sent holiday postcards to each other. How many postcards have been sent?

e) How many elements does a set of all five-digit natural numbers contain?

f) The shop offers seven kinds of postcards. In how many ways can be bought

i) ten postcards,

ii) five postcards,

iii) five different postcards?

g) Specify the number of all possible tosses of

i) two six-sided dice,

ii) three six-sided dice. 


\section{Basic probability}

\subsection{Random trial, random event}

Random trial - any action with a random result (its outcome is not known in advance)

Sample space $\Omega$ - the set of all possible outcomes of a random trial

Random event $A$ - any subset of $\Omega, A \subset \Omega$

Impossible event - event $A$ that never occurs, $A=\varnothing$

Sure event - event $A$ that always occurs, $A=\Omega$

Complementary (oposit) event - event $\bar{A}$ that occurs in case $A$ does not occur, $\bar{A}=\Omega-A$

Example 5

Random trial:

throwing a regular die

Sample space $\Omega$ :

the set of all possible outcomes $\Omega=\{1,2,3,4,5,6\}$

Random event:

$A$... the result is odd

$A=\{3,5,7\}$

Impossible event:

$B$... the result is less than 1

$B=\varnothing$

Sure event:

$C$... the result is larger than 0

$C=\Omega$

Complement of $A$ :

$\bar{A}$... the result is even

$\bar{A}=\{2,4,6\}$

\section{Relationship between events}

Event $A$ is a subset of event $B$ (if $A$ occurs then $B$ occurs):

$$
A \subset B \Leftrightarrow\{\forall \omega \in \Omega:(\omega \in A) \Rightarrow(\omega \in B)\}
$$

Events $A$ and $B$ are equal ( $A$ occurs if and only if $B$ occurs):

$$
A=B \Leftrightarrow\{\forall \omega \in \Omega:(\omega \in A) \Leftrightarrow(\omega \in B)\}
$$

Events $A$ and $B$ are mutually exclusive or disjoint $\Leftrightarrow$ they cannot happen at the same time. Events $A_{1}, A_{2}, \ldots, A_{n}$ are mutually exclusive $\Leftrightarrow$ the occurrence of any one of them implies the non-occurrence of the remaining $n-1$ events.

Events $A_{1}, A_{2}, \ldots, A_{n}$ are jointly or collectively exhaustive $\Leftrightarrow$ at least one of them must occur.

Events $A_{1}, A_{2}, \ldots, A_{n}$ partition the sample space $\Omega \Leftrightarrow$ they are mutually exclusive and collectively exhaustive.

\section{Operations with events}

The union of $A$ and $B$ ( the occurrence of $A$ or $B$ or both, " $A$ or $B$ "):

$A \cup B=\{\forall \omega \in \Omega:(\omega \in A) \vee(\omega \in B)\}$

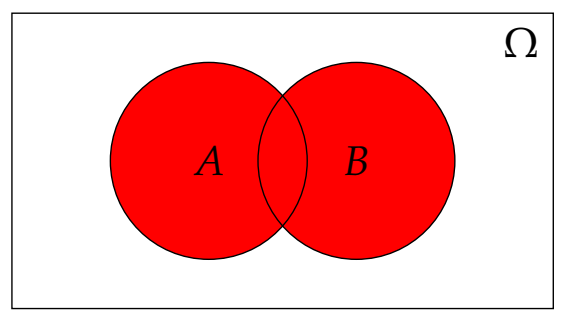


The intersection of $A$ and $B$ (the occurrence of both $A$ and $B$, " $A$ and $B$ "): $A \cap B=\{\forall \omega \in \Omega:(\omega \in A) \wedge(\omega \in B)\}$

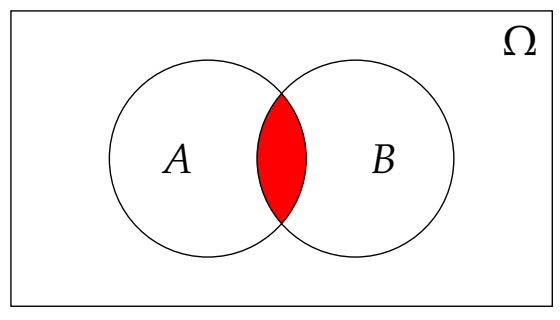

The difference of $A$ and $B$ (the occurrence of $A$ but not $B$, " $A$ but not $B$ "):

$A-B=\{\forall \omega \in \Omega:(\omega \in A) \wedge(\omega \notin B)\}$

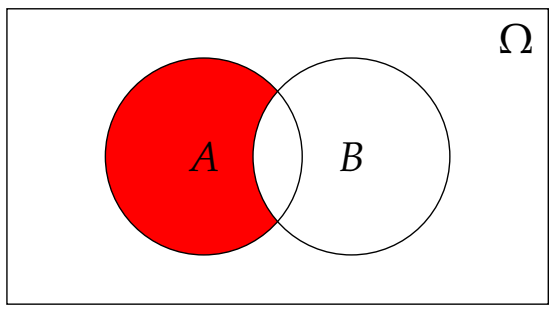

The complement of $A$ (the non-occurrence of $A$, "not $A$ "):

$\bar{A}=\{\forall \omega \in \Omega: \omega \notin A\}$

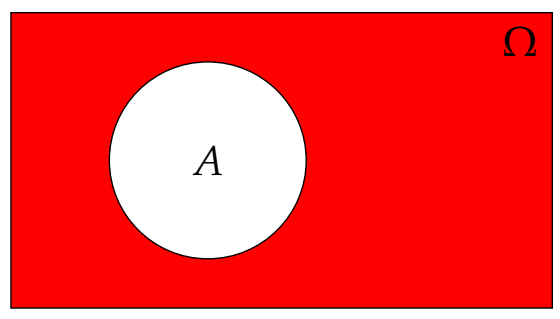

Example 6

A player throws two fair dice (the first one is red and the second one is blue). Write out all the elements of the sample space $\Omega$ and of the following random events:

$A_{1} \ldots$ the number of dots on the face of the red die equals six

$A_{2} \ldots$ the number of dots on the face of the blue die equals one

$A_{3} \ldots$ the number of dots on the face of the red die equals six and the number of dots on the face of the blue die equals one

$A_{4} \ldots$ the number of dots on the face of the red die equals six or the number of dots on the face of the blue die equals one

$A_{5} \ldots$ the number of dots on the face of the red die equals six and the number of dots on the face of the blue die does not equal one

$A_{6} \ldots$ the number of dots on the face of the red die does not equal six

$A_{7} \ldots$ the sum of dots on the faces of the red and blue die is less than or equal to twelve

$A_{8} \ldots$ the sum of dots on the faces of the red and blue die is greater than twelve

$A_{9} \ldots$ the numbers of dots on the faces of both dice is even

$A_{10} \ldots$ the numbers of dots on the faces of both dice is odd

$A_{11} \ldots$ one of the numbers of dots on the faces of dice is even and the second one is odd 


$$
\begin{aligned}
& \Omega=\{(1,1),(1,2),(1,3),(1,4),(1,5),(1,6), \\
& (2,1),(2,2),(2,3),(2,4),(2,5),(2,6) \text {, } \\
& (3,1),(3,2),(3,3),(3,4),(3,5),(3,6) \text {, } \\
& (4,1),(4,2),(4,3),(4,4),(4,5),(4,6) \text {, } \\
& (5,1),(5,2),(5,3),(5,4),(5,5),(5,6) \text {, } \\
& (6,1),(6,2),(6,3),(6,4),(6,5),(6,6)\} \\
& A_{1}=\{(6,1),(6,2),(6,3),(6,4),(6,5),(6,6)\} \\
& A_{2}=\{(1,1),(2,1),(3,1),(4,1),(5,1),(6,1)\} \\
& A_{3}=A_{1} \cap A_{2}=\{(6,1)\} \\
& A_{4}=A_{1} \cup A_{2}=\{(6,1),(6,2),(6,3),(6,4),(6,5),(6,6),(1,1),(2,1),(3,1),(4,1),(5,1)\} \\
& A_{5}=A_{1}-A_{2}=\{(6,2),(6,3),(6,4),(6,5),(6,6)\} \\
& A_{6}=\bar{A}_{1}=\{(1,1),(1,2),(1,3),(1,4),(1,5),(1,6) \text {, } \\
& (2,1),(2,2),(2,3),(2,4),(2,5),(2,6) \text {, } \\
& (3,1),(3,2),(3,3),(3,4),(3,5),(3,6) \text {, } \\
& (4,1),(4,2),(4,3),(4,4),(4,5),(4,6) \text {, } \\
& (5,1),(5,2),(5,3),(5,4),(5,5),(5,6)\} \\
& A_{7}=\Omega \\
& A_{8}=\varnothing \\
& A_{9}=\{(2,2),(2,4),(2,6),(4,2),(4,4),(4,6),(6,2),(6,4),(6,6)\} \\
& A_{10}=\{(1,1),(1,3),(1,5),(3,1),(3,3),(3,5),(5,1),(5,3),(5,5)\} \\
& A_{11}=\{(1,2),(1,4),(1,6),(2,1),(2,3),(2,5),(3,2),(3,4),(3,6) \text {, } \\
& (4,1),(4,3),(4,5),(5,2),(5,4),(5,6),(6,1),(6,3),(6,5)\}
\end{aligned}
$$

\subsection{Probability of random event}

\section{Definition}

The probability of random event $A, A \subset \Omega$, is defined by the formula:

$$
P(A)=\frac{m}{n}
$$

where $n$ is the number of all possible outcomes of a random trial and $m$ is the number of all outcomes of a random trial that meet the specification of $A$.

Theorem (Probability characteristics)

1. $P(\varnothing)=0, P(\Omega)=1$

2. $P(\bar{A})=1-P(A)$

3. $P(A \cup B)=P(A)+P(B)-P(A \cap B)$

Example 7

Of 20 light bulbs, 7 are defective. A group of 5 bulbs is chosen at random. Calculate the probability that:

a) none of them is defective.

b) two of them are defective. 
c) at least one of them is defective.

a) Choosing a group of 5 bulbs from a group of 20 we make combinations without repetition. Their number equals $C_{5}(20)$, which is the number of all possible results of our random trial. The number of all outcomes that meet the specification of a) equals $C_{5}$ (13) (0 defective means 5 non-defective bulbs, their number is $20-7=13$ ).

$$
P(A)=\frac{C_{5}(13)}{C_{5}(20)}=\frac{\left(\begin{array}{c}
13 \\
5
\end{array}\right)}{\left(\begin{array}{c}
20 \\
5
\end{array}\right)} \doteq 0.0830
$$

b) The number of all outcomes that meet the specification of b) equals $C_{2}(7) \cdot C_{3}(13)$ ( 2 defective means 3 non-defective bulb).

$$
P(B)=\frac{C_{2}(7) \cdot C_{3}(13)}{C_{5}(20)}=\frac{\left(\begin{array}{c}
7 \\
2
\end{array}\right) \cdot\left(\begin{array}{c}
13 \\
3
\end{array}\right)}{\left(\begin{array}{c}
20 \\
5
\end{array}\right)} \doteq 0.3874
$$

c) Random event $\mathrm{C}$ is the complement of random event $\mathrm{A}$.

Exercise 8

$$
P(C)=1-P(A) \doteq 0.9170
$$

a) If three balls are drawn at random from a bag containing 6 red balls, 4 white balls and 8 blue balls, what is the probability that all three are red?

b) A shipment of 17 radios includes 5 radios that are defective. The receiver samples 6 radios at random. What is the probability that exactly 3 of the selected radios are defective?

c) Three married couples have purchased theater tickets and are seated in a row consisting of just six seats. If they take their seats in a completely random fashion, what is the probability that

i) Jim and Paula (husband and wife) sit in the two seats on the far left.

ii) Jim and Paula end up sitting next to one another.

d) An experiment consists of rolling two dice simultaneously and independently of one another. Find the probability of the event consisting of having an odd number in the first roll or a total of 9 in both rolls.

\subsection{Conditional probability, independent events}

\section{Definition}

The conditional probability of $A$ given that $B$ occurs, or on condition that $B$ occurs, is defined by the formula:

$$
P(A \mid B)= \begin{cases}\frac{P(A \cap B)}{P(B)} & \text { if } P(B) \neq 0 \\ 0 & \text { if } P(B)=0\end{cases}
$$


Definition

The events $A$ and $B$ are independent $\Leftrightarrow P(A)=P(A \mid B)$.

Remark

- Two events are statistically independent when the occurrence of one has no influence on the occurrence of the other.

- $P(A \cap B)=P(A \mid B) \cdot P(B) \ldots$ for any random events $A, B$

- $P(A \cap B)=P(A) \cdot P(B) \quad \ldots$ for independent random events $A, B$

- $P(A \cap B)=P(A) \cdot P(B) \quad \Leftrightarrow \quad$ random events $A$ and $B$ are independent

\section{Definition}

The events $A_{1}, A_{2}, \ldots, A_{n}$ are mutually independent $\Leftrightarrow$

$$
P\left(\bigcap_{i \in M} A_{i}\right)=\prod_{i \in M} P\left(A_{i}\right), \forall M \subset\{1,2, \ldots, n\} .
$$

\section{Example 9}

If 2 balls are drawn at random from a box with 5 white and 7 black balls, what is the probability that both two balls are white? Suppose that the first drawn ball

a) is put back before the second ball is drawn.

b) is not put back before the second ball is drawn.

Consider the following three events:

$A_{i} \ldots i$-th ball is white, $i=1,2$

$A$... both balls are white $\left(A=A_{1} \cap A_{2}\right)$

a) $A_{1}$ and $A_{2}$ are independent (as the results of the first and second draws are independent of one another), so we use the formula for independent events $P(A \cap B)=$ $P(A) \cdot P(B)$ for computation:

$$
P(A)=P\left(A_{1} \cap A_{2}\right)=P\left(A_{1}\right) \cdot P\left(A_{2}\right)=\frac{5}{12} \cdot \frac{5}{12} \doteq 0.1736
$$

b) $A_{1}$ and $A_{2}$ are not independent (as the result of the second draw depends on the result of the first one), so we use the formula for dependent events $P(A \cap B)=P(A \mid B)$. $P(B)$ for computation:

$$
P(A)=P\left(A_{1} \cap A_{2}\right)=P\left(A_{1}\right) \cdot P\left(A_{2} \mid A_{1}\right)=\frac{5}{12} \cdot \frac{4}{11} \doteq 0.1515
$$


Exercise 10

a) Three shooters shoot independently to the same target. The probabilities of their hits are 0.8, 0.7 and 0.6. Each shooter shoots one shot. What is the probability that the target will be hit by

i) all of them,

ii) non of them,

iii) at least one of them,

iv) exactly one of them?

b) The probabilities of the monthly snowfall exceeding $10 \mathrm{~cm}$ at a particular location in the months of December, January, and February are 0.2, 0.4, and 0.6, respectively. For a particular winter, what is the probability

i) that the snowfall will be less than $10 \mathrm{~cm}$ in all the 3 months,

ii) of receiving at least $10 \mathrm{~cm}$ snowfall in at least 2 of the 3 months?

\subsection{The total probability theorem, Bayes' theorem}

\section{Definition}

The collection of sets $\left\{A_{1}, A_{2}, \ldots, A_{n}\right\}$ partitions the sample space $\Omega \Leftrightarrow$ the events $A_{1}, A_{2}, \ldots, A_{n}$ are mutually exclusive and collectively exhaustive.

\section{Theorem (Total probability theorem)}

If the collection of sets $\left\{B_{1}, B_{2}, \ldots, B_{n}\right\}$ partitions the sample space $\Omega$, then the following formula is valid for any set $A$ in the sample space $\Omega$ :

$$
P(A)=\sum_{i=1}^{n} P\left(A \mid B_{i}\right) \cdot P\left(B_{i}\right)
$$

\section{- Theorem (Bayes' theorem)}

If the collection of sets $\left\{B_{1}, B_{2}, \ldots, B_{n}\right\}$ partitions the sample space $\Omega$, then the following formula is valid for any set $A$ in the sample space $\Omega$ :

$$
P\left(B_{k} \mid A\right)=\frac{P\left(A \mid B_{k}\right) \cdot P\left(B_{k}\right)}{\sum_{i=1}^{n} P\left(A \mid B_{i}\right) \cdot P\left(B_{i}\right)}
$$

\section{Example 11}

It is known that of the articles produced by a factory, $20 \%$ come from Machine 1, 30 $\%$ from Machine 2, and $50 \%$ from Machine 3. The percentages of satisfactory articles among those produced are $95 \%$ for Machine 1, $85 \%$ for Machine 2 and $90 \%$ for Machine 3 . An article is chosen at random.

a) What is the probability that it is satisfactory?

b) Assuming that the article is satisfactory, what is the probability that it was produced by Machine 1? 
Consider the following events $B_{1}, B_{2}, B_{3}$ and $A$ :

$B_{i} \ldots$ the $i$-th article is produced by Machine $i, i=1, \ldots, 3$

$A$... the article is satisfactory

The following probabilities are known from the text:

$P\left(B_{1}\right)=0.20, P\left(B_{2}\right)=0.30, P\left(B_{3}\right)=0.50$

$P\left(A \mid B_{1}\right)=0.95, P\left(A \mid B_{2}\right)=0.85, P\left(A \mid B_{3}\right)=0.90$

a) The formula from the total probability theorem is used to solve question a):

$$
P(A)=\sum_{i=1}^{3} P\left(A \mid B_{i}\right) \cdot P\left(B_{i}\right)=0.20 \cdot 0.95+0.30 \cdot 0.85+0.50 \cdot 0.90=0.8950
$$

b) The formula from Bayes' theorem is used to solve question b):

$$
P\left(B_{1} \mid A\right)=\frac{P\left(A \mid B_{1}\right) \cdot P\left(B_{1}\right)}{\sum_{i=1}^{3} P\left(A \mid B_{i}\right) \cdot P\left(B_{i}\right)}=\frac{0.20 \cdot 0.95}{0.20 \cdot 0.95+0.30 \cdot 0.85+0.50 \cdot 0.90} \doteq 0.2123
$$

Exercise 12

a) An $80 \%$ of people attend their primary care physician regularly; $35 \%$ of those people have no health problems crop up during the following year. Out of the 20 $\%$ of people who do not see their doctor regularly, only $5 \%$ have no health issues during the following year. What is the probability a random person will have no health problems in the following year?

b) In a certain county: $60 \%$ of registered voters are Republicans, $30 \%$ are Democrats and $10 \%$ are Independents. When those voters were asked about increasing military spending: $40 \%$ of Republicans opposed it, $65 \%$ of the Democrats opposed it and $55 \%$ of the Independents opposed it.

i) What is the probability that a randomly selected voter opposes increased military spending?

ii) A registered voter from our county writes a letter to the local paper, arguing against increased military spending. What is the probability that this voter is a Democrat? 


\section{Random variable}

\section{Definition}

A random variable $X$ is a function $X: \Omega \rightarrow \mathbb{R}$ that assigns a number to every outcome of a random experiment.

We denote random variables by capital letters $X, Y, \ldots$ and their particular values by small letters $x, y, \ldots$

The range of a random variable $X$, denoted by $R_{X}$ or $M$, is the set of possible values for $X$.

There can be two types of random variables depending on the possible set of values.

- Discrete random variable - the possible set of values is finite or countable

- Continuous random variable - the possible set of values is a range (closed or open interval, not discrete values)

\subsection{Distribution function}

All random variables have a cumulative distribution function. It is a function giving the probabilities that the random variable $X$ is less than $x$, for every value $x$.

\section{Definition}

A cumulative distribution function $F(x)$ of the random variable $X$ is defined as

$$
F(x)=P(X<x)
$$

for $x \in \mathbb{R}$.

Some important properties of $F(x)$ :

- $0 \leq F(x) \leq 1$

- $P\left(x_{1} \leq X<x_{2}\right)=F\left(x_{2}\right)-F\left(x_{1}\right)$

- $F(x)$ is a non-decreasing function:

$$
\forall x_{1}, x_{2} \in \mathbb{R}: x_{1}<x_{2} \Rightarrow F\left(x_{1}\right) \leq F\left(x_{2}\right)
$$

- $F(x)$ is a left-continuous function:

$$
\forall a \in \mathbb{R}: \lim _{x \rightarrow a^{-}} F(x)=F(a)
$$

- it has limits

$$
\lim _{x \rightarrow-\infty} F(x)=0, \quad \lim _{x \rightarrow \infty} F(x)=1
$$


Remark

In the definition, the "less than" sign, " $<$ ", is a convention, not an universally used one. The distribution function can be define as "less than or equal to" sign, " $\leq$ ". Then the distribution function is defined as right-continuous function: $F(x)=P(X \leq x)$.

\subsection{Probability function}

The probability distribution of a discrete random variable is a list of probabilities associated with each of its possible values.

\section{Definition}

Let $X$ be a discrete random variable with range $R_{X}$. Probability mass function $p(x)$ is defined as

$$
p(x)=P(X=x)
$$

for $x \in R_{X}$.

Some properties of $p(x)$ :

- $p(x) \geq 0$ for all values from $\mathbb{R}$

- the sum of $p(x)$ over all possible values of $x$ is 1

$$
\sum_{i=1}^{k} p\left(x_{i}\right)=1 \text {, where } k \text { is the maximum possible value of } i
$$

- cumulative probabilities are found by adding individual probabilities $p\left(x_{i}\right)$

$$
F(x)=P(X<x)=\sum_{x_{i}<x} p\left(x_{i}\right)
$$

Example 13

There are two coffee machines in the theater foyer. The probability of failure is $7 \%$ for the first machine and $5 \%$ for the second machine. The random variable represents the number of broken machines. Find the probability and the distribution function of the given random variable.

$X=$ the number of broken machines

$X$ can take three values $\{0,1,2\} \Rightarrow X$ is discrete random variable

Consider the following events: $A_{i} \ldots$ the $i$-th machine is out of order

The probabilities of the events $A_{i}$ are known from the text

$$
\begin{aligned}
& P\left(A_{1}\right)=0.07 \\
& P\left(A_{2}\right)=0.05
\end{aligned}
$$


The probability function is:

$p(0)=P(X=0)=P\left(\bar{A}_{1}\right) \cdot P\left(\bar{A}_{2}\right)=0.93 \cdot 0.95=0.8835$

$p(1)=P(X=1)=P\left(\bar{A}_{1}\right) \cdot P\left(A_{2}\right)+P\left(A_{1}\right) \cdot P\left(\bar{A}_{2}\right)=0.93 \cdot 0.05+0.07 \cdot 0.95=0.113$

$p(2)=P(X=2)=P\left(A_{1}\right) \cdot P\left(A_{2}\right)=0.07 \cdot 0.05=0.0035$

\begin{tabular}{c|ccc}
\hline$x$ & 0 & 1 & 2 \\
\hline$p(x)$ & 0.8835 & 0.113 & 0.0035 \\
\hline
\end{tabular}

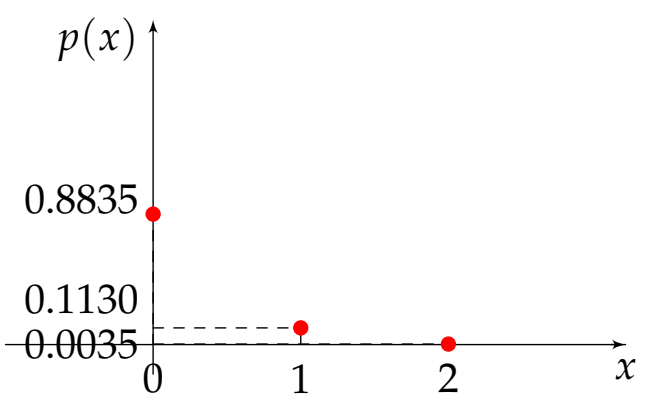

We calculate values of the distribution function

$\forall x \in(-\infty ; 0]: F(x)=P(X<x)=0$

$\forall x \in(0 ; 1]: F(x)=P(X<x)=P(X=0)=0.8835$

$\forall x \in(1 ; 2]: F(x)=P(X<x)=P(X=0)+P(X=1)=0.8835+0.113=0.9965$

$\forall x \in(2 ; \infty): F(x)=P(X<x)=P(X=0)+P(X=1)+P(X=2)=0.8835+0.113+$ $0.0035=1$

\begin{tabular}{c|cccc}
\hline$x \in$ & $(-\infty, 0]$ & $(0,1]$ & $(1,2]$ & $(2, \infty)$ \\
\hline$F(x)$ & 0 & 0.8835 & 0.9965 & 1 \\
\hline
\end{tabular}

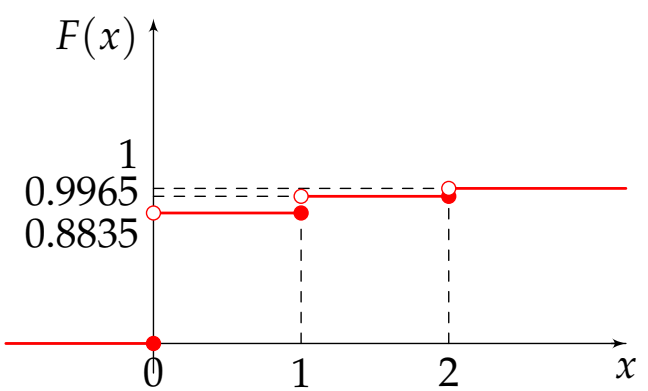

\section{Exercise 14}

a) The shooter has 3 bullets and shoots at the target until the first hit or until the last bullet. The probability that the shooter hits the target after one shot is 0.7 . The random variable $X$ is the number of the fired bullets. Find the probability and the distribution function of the given random variable. What is the probability that the number of the fired bullets will not be larger then 2 ?

b) Toss a coin 3 times. Let $X$ be the number of heads observed. Find the probability and the distribution function of the given random variable. 


\subsection{Probability density function}

Because for a continuous random variable $P(X=x)=0$ for all $x \in \mathbb{R}$, the probability mass function does not work for continuous random variables. Instead, we can usually define the probability density function. The continuous random variable is represented by the area under a curve (this is known as an integral).

\section{Definition}

The probability density function (pdf) of the random variable $X$ is non-negative function $f(x)$ such that

$$
f(x)=\lim _{h \rightarrow 0} \frac{P(x \leq X<x+h)}{h}
$$

for $x \in[a, b]$.

Some properties of $f(x)$ :

- $\forall x \in \mathbb{R}: f(x) \geq 0$

- $f(x)=F^{\prime}(x) ; \quad F(x)=\int_{-\infty}^{x} f(x) \mathrm{d} x$

- $\lim _{x \rightarrow \infty} f(x)=0, \lim _{x \rightarrow-\infty} f(x)=0$

- $\int_{-\infty}^{\infty} f(x)=1$

- $P\left(x_{1} \leq X<x_{2}\right)=F\left(x_{2}\right)-F\left(x_{1}\right)=\int_{x_{1}}^{x_{2}} f(x) \mathrm{d} x$

Example 15

The random variable $X$ has the probability density function

$$
f(x)=\left\{\begin{array}{ll}
C \mathrm{e}^{-2 x} & \text { pro } 0<x<2 \\
0 & \text { otherwise }
\end{array} .\right.
$$

Determine a constant $C$ in order that $f(x)$ is a probability density function. Calculate the probability $P(X<1)$.

The probability density function has to fulfill

$$
\int_{-\infty}^{\infty} f(x) \mathrm{d} x=1 \Rightarrow \int_{-\infty}^{0} 0 \mathrm{~d} x+\int_{0}^{2} C \mathrm{e}^{-2 x} \mathrm{~d} x+\int_{0}^{\infty} 0 \mathrm{~d} x=1
$$

So

$$
\int_{0}^{2} C \mathrm{e}^{-2 x} \mathrm{~d} x=1 \Rightarrow C \int_{0}^{2} \mathrm{e}^{-2 x} \mathrm{~d} x=C\left[\frac{\mathrm{e}^{-2 x}}{-2}\right]_{0}^{2}=C\left(\frac{\mathrm{e}^{-4}}{-2}+\frac{1}{2}\right)=1 \Rightarrow C \cdot \frac{1-\mathrm{e}^{-4}}{2}=1,
$$


we get $\quad C=\frac{2}{1-\mathrm{e}^{-4}} \doteq 2.04$

Using the pdf we can calculate

$$
P(X<1)=\int_{0}^{1} \frac{2}{1-\mathrm{e}^{-4}} \cdot \mathrm{e}^{-2 x} \mathrm{~d} x=\frac{2}{1-\mathrm{e}^{-4}}\left[\frac{\mathrm{e}^{-2 x}}{-2}\right]_{0}^{1}=\frac{2}{1-\mathrm{e}^{-4}}\left(\frac{\mathrm{e}^{-2}}{-2}+\frac{1}{2}\right) \doteq 0.88
$$

Exercise 16

A probability density function is given by

$$
f(x)= \begin{cases}0 & x<1 \\ b / x^{2} & x \in[1 ; 5] \\ 0 & x>5\end{cases}
$$

a) What is the value of $b$ ?

b) From this obtain the probability that $X$ is between 2 and 4 .

c) What is the probability that $X$ is exactly 2 ?

d) Find the cumulative distribution function of $X$.

\subsection{Numerical characteristics}

The distribution function (the probability function or the probability density function) gives us the complete information about the random variable. Sometimes it is useful to know some simpler and concentrated formulation of this information such as measures of location, dispersion and concentration.

\section{Definition}

Let $X$ be a random variable. The expectation $E(X)$ of $X$ (the mean $\mu$ of $X$ ) is defined by

- $\mu \equiv E(X)=\sum_{i} x_{i} \cdot p\left(x_{i}\right)$, if $X$ is a discrete random variable

- $\mu \equiv E(X)=\int_{-\infty}^{\infty} x \cdot f(x) \mathrm{d} x$, if $X$ is a continuous random variable

\section{Properties:}

- $E(c)=c, \quad c \in \mathbb{R}$

- $E(a X+b)=a E(X)+b \quad$ for all $a, b \in \mathbb{R}$

- $E\left(X_{1}+X_{2}+\cdots+X_{n}\right)=E\left(X_{1}\right)+E\left(X_{2}\right)+\cdots+E\left(X_{n}\right)$ for any set of random variables

- if $X_{1}, X_{2}, \ldots, X_{n}$ are independent: $E\left(X_{1} \cdot X_{2} \cdots X_{n}\right)=E\left(X_{1}\right) \cdot E\left(X_{2}\right) \cdots E\left(X_{n}\right)$ 


\section{Definition}

Let $X$ be a random variable. The variance of $X, \operatorname{var}(X), D(X)$ or $\sigma^{2}$ is given by

- $\sigma^{2} \equiv \operatorname{var}(X)=\sum_{i}\left(x_{i}-\mu\right)^{2} \cdot p\left(x_{i}\right)$, if $X$ is a discrete random variable

- $\sigma^{2} \equiv \operatorname{var}(X)=\int_{-\infty}^{\infty}(x-\mu)^{2} \cdot f(x) \mathrm{d} x$, if $X$ is a continuous random variable

The variance is a measure of how spread out the distribution of a random variable is.

The variance of a random variable $X$ can be also defined as

$$
\operatorname{var}(X)=E\left(X^{2}\right)-(E(X))^{2} .
$$

\section{Properties:}

- $\operatorname{var}(c)=0, \quad c \in \mathbb{R}$

- $\operatorname{var}(a X+b)=a^{2} \operatorname{var}(X)$ for all $a, b \in \mathbb{R}$

- $\operatorname{var}(X+Y)=\operatorname{var}(X)+\operatorname{var}(Y)$, if $X$ and $Y$ are independent

\section{Definition}

The standard deviation $\sigma$ of a random variable $X$ is defined as

$$
\sigma=\sqrt{\operatorname{var}(X)}
$$

The variance and the standard deviation are non-negative. The standard deviation of $X$ has the same unit as $X$, but the variance has a different unit than $X$ - unit ${ }^{2}$.

\section{Definition}

The $r$-th central moment $\mu_{r}$ is defined by

- $\mu_{r}=\sum_{i}\left(x_{i}-\mu\right)^{r} \cdot p\left(x_{i}\right)$, if $X$ is a discrete random variable.

- $\mu_{r}=\int_{-\infty}^{\infty}(x-\mu)^{r} \cdot f(x) \mathrm{d} x$, if $X$ is a continuous random variable.

\section{Definition}

The skewness $\mathrm{a}_{3}, A$ is defined by

$$
A \equiv \mathrm{a}_{3}=\frac{\mu_{3}}{\sigma^{3}} \text {, where } \mu_{3} \text { is the } 3 \text { rd central moment of } X \text {. }
$$



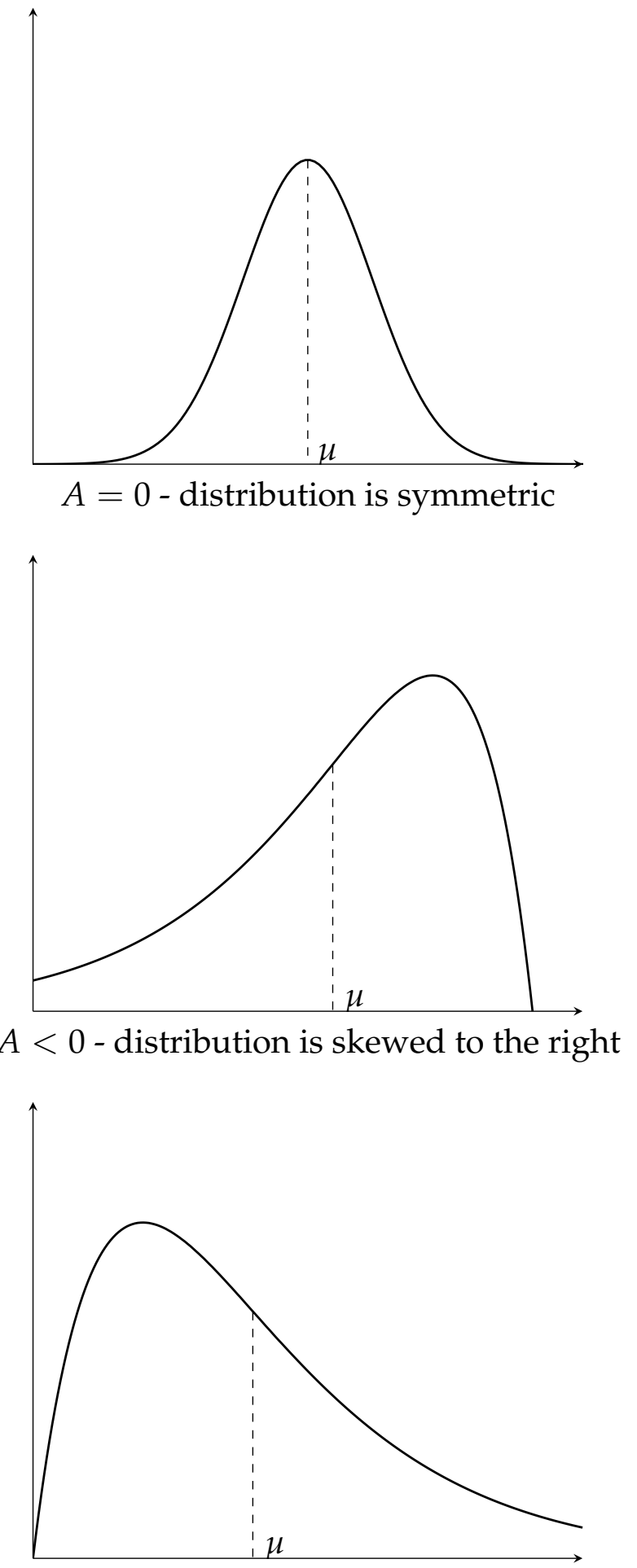

$A>0$ - distribution is skewed to the left

\section{Definition}

The kurtosis $\mathrm{a}_{4}, e$ is defined by

$$
e \equiv \mathrm{a}_{4}=\frac{\mu_{4}}{\sigma^{4}} \text {, where } \mu_{4} \text { is the } 4 \text { th central moment of } X \text {. }
$$

The kurtosis is a measure of whether the data are heavy-tailed or light-tailed relative to a normal distribution. The standard normal distribution has a kurtosis equal to 3 . Data sets 
with high kurtosis tend to have heavy tails, or outliers. Data sets with low kurtosis tend to have light tails, or lack of outliers. Some sources use the excess kurtosis $\bar{e}=e-3$.

\section{The quantiles}

The quantiles are the values which divide the distribution such that there is a given proportion of observations below the quantile.

\section{Definition}

The $p$-quantile $x_{p}$ of a random variable $X$ is such a value of $X$ that

$$
F\left(x_{p}\right)=p,
$$

where $0<p<1$.

The median $x_{0.5}$ is the central value of the distribution, such that half the values are less than or equal to it and half are greater than or equal to it

$$
P\left(X \leq x_{0.5}\right)=P\left(X \geq x_{0.5}\right)=0.5 \text {. }
$$

The quartiles divide the distribution into four equal parts, called fourths. The first quartile $x_{0.25}$ is 0.25 -quantile, the second quartile is the median (0.5-quantile) and the third quartile $x_{0.75}$ is 0.75 -quantile.

\section{The mode}

- a discrete random variable $X$ - the mode is the value with the greatest probability (the value $x$ at which $P(X=x)$ reaches a maximum), it is the value of $X$ that is most likely to occur. Distributions with only one maximum are called unimodal, those with two maxima bimodal.

- a continuous random variable - the mode is the point at which the probability density function reaches a local maximum, or a peak. It is not the value of $X$ most likely to occur.

\section{Example 17}

A discrete random variable $X$ has the following probability distribution table

\begin{tabular}{c|cccc}
\hline$x$ & 0 & 2 & 3 & 5 \\
\hline$P(X=x)$ & $\frac{1}{7}$ & $\frac{1}{7}$ & $\frac{3}{7}$ & $\frac{2}{7}$ \\
\hline
\end{tabular}

Find $F(x)$. Calculate the numerical characteristics of this random variable.

\section{The distribution function}

\begin{tabular}{c|ccccc}
\hline$X$ & $(-\infty, 0]$ & $(0,2]$ & $(2,3]$ & $(3,5]$ & $(5, \infty)$ \\
\hline$F(x)$ & 0 & $\frac{1}{7}$ & $\frac{2}{7}$ & $\frac{5}{7}$ & 1 \\
\hline
\end{tabular}


The expected value

$E(X)=\sum_{i=1}^{4} x_{i} p\left(x_{i}\right)=0 \cdot \frac{1}{7}+2 \cdot \frac{1}{7}+3 \cdot \frac{3}{7}+5 \cdot \frac{2}{7}=\frac{2+9+10}{7}=\frac{21}{7}=3$

The variance + the standard deviation

$D(X)=E\left(X^{2}\right)-[E(X)]^{2}=\sum_{i=1}^{4} x_{i}^{2} p\left(x_{i}\right)-[E(X)]^{2}=0^{2} \cdot \frac{1}{7}+2^{2} \cdot \frac{1}{7}+3^{2} \cdot \frac{3}{7}+5^{2} \cdot \frac{2}{7}-3^{2}=$ $\frac{81}{7}-9 \doteq 2.57$

$\sigma=\sqrt{D(X)}=\sqrt{2.57} \doteq 1.6$

The skewness + the kurtosis

$\mathrm{a}_{3}=\frac{\sum_{i=1}^{4}\left(x_{i}-\mu\right)^{3} p\left(x_{i}\right)}{\sigma^{3}} \doteq \frac{(0-3)^{3} \cdot \frac{1}{7}+(2-3)^{3} \cdot \frac{1}{7}+(3-3)^{3} \cdot \frac{3}{7}+(5-3)^{3} \cdot \frac{2}{7}}{1.6^{3}} \doteq-0.42$

$\mathrm{a}_{4}=\frac{\sum_{i=1}^{4}\left(x_{i}-\mu\right)^{4} p\left(x_{i}\right)}{\sigma^{4}} \doteq \frac{(0-3)^{4} \cdot \frac{1}{7}+(2-3)^{4} \cdot \frac{1}{7}+(3-3)^{4} \cdot \frac{3}{7}+(5-3)^{4} \cdot \frac{2}{7}}{1.6^{4}} \doteq 2.46$

\section{The mode}

Looking at the probability table we can see that the greatest probability is $P(X=3)=\frac{3}{7}$. So the mode is 3 .

Exercise 18

A discrete random variable $X$ has the following probability distribution table

\begin{tabular}{c|ccc}
\hline$x$ & 1 & 2 & 3 \\
\hline$P(X=x)$ & $\frac{1}{6}$ & $\frac{1}{3}$ & $\frac{1}{2}$ \\
\hline
\end{tabular}

a) Show this probability function as a graph.

b) Sketch a graph of the corresponding cumulative distribution function.

c) Find the expected value.

d) Find the standard deviation and the skewness.

e) Find the mode. 


\section{Discrete probability distribution}

\section{Discrete uniform distribution $U(n)$}

All values of the random variable $n$ have the same constant probability. Uniform means that each of the values is equally likely.

$$
\begin{gathered}
p(x)=P(X=x)=\frac{1}{n}, \quad x=1,2, \ldots, n \\
E(X)=\mu=\frac{n+1}{2}, D(X)=\sigma^{2}=\frac{n^{2}-1}{12}
\end{gathered}
$$

Typical application: Rolling one die

The possible outcomes are $\{1,2,3,4,5,6\}$, each with probability $\frac{1}{6}$. The number of possible outcomes is $n=6 \Rightarrow$ the outcomes of the roll of a fair die form an uniform distribution $U(6)$.

The expected value (mean): $\mu=\frac{6+1}{2}=3.5$

The variance: $\sigma^{2}=\frac{6^{2}-1}{12}=\frac{35}{12} \doteq 2.9$ and the standard deviation: $\sigma=\sqrt{\frac{35}{12}} \doteq 1.7$

Probability table:

\begin{tabular}{l|llllll}
\hline$x_{i}$ & 1 & 2 & 3 & 4 & 5 & 6 \\
\hline$p\left(x_{i}\right)$ & $\frac{1}{6}$ & $\frac{1}{6}$ & $\frac{1}{6}$ & $\frac{1}{6}$ & $\frac{1}{6}$ & $\frac{1}{6}$ \\
\hline
\end{tabular}

Graphical representation:
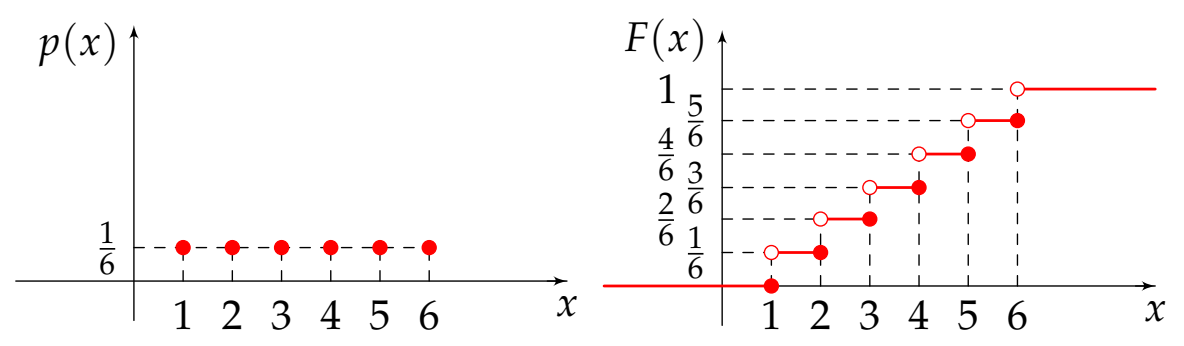

The probability that an odd number on the top of the die is

$$
P(X=\text { odd number })=P(X=1)+P(X=3)+P(X=5)=\frac{1}{6}+\frac{1}{6}+\frac{1}{6}=0.5
$$

Binomial distribution $B i(n, p)$

$$
p(x)=\left(\begin{array}{l}
n \\
x
\end{array}\right) p^{x}(1-p)^{n-x}, \quad x=0,1, \ldots, n,
$$

where $n$ is the number of independent trials and $p$ is the probability of success in each trial. 
$E(X)=n p, D(X)=n p(1-p)$

Excel:

$p(x)=P(X=x)=\operatorname{BINOM}$.DIST $(x ; n ; p ; 0)$

$F(x)=P(X \leq x)=\operatorname{BINOM}$.DIST $(x ; n ; p ; 1)$

\section{Example 19}

Suppose you independently throw a dart 10 times. Each time you throw a dart, the probability of hitting the target is $\frac{1}{3}$. What is the probability of hitting the target three times?

Let $X$ denote the number of times you hit. The possible values of $X$ are $\{0,1, \ldots, 10\} \Rightarrow$ $X \sim B i\left(10 ; \frac{1}{3}\right)$.

The probability of hitting the target 3 times can be computed from the probability function of $X$ :

$$
P(X=3)=p(3)=\left(\begin{array}{c}
10 \\
3
\end{array}\right) \cdot\left(\frac{1}{3}\right)^{3} \cdot\left(\frac{2}{3}\right)^{7}
$$

Excel:

$$
P(X=3)=\text { BINOM.DIST }\left(3 ; 10 ; \frac{1}{3} ; 0\right) \doteq 0.26
$$

Hypergeometric distribution $H(n, M, N)$

$$
p(x)=\frac{\left(\begin{array}{c}
M \\
x
\end{array}\right)\left(\begin{array}{c}
N-M \\
n-x
\end{array}\right)}{\left(\begin{array}{c}
N \\
n
\end{array}\right)}, \quad x=\max \{0, M-N+n\} \ldots \min \{M, n\},
$$

where $n$ is the number of trials, $N$ is the number of units in the population and $M$ is the number in the population classified as success.

$E(X)=\frac{M n}{N}, D(X)=\frac{M n(N-M)(N-n)}{N^{2}(N-1)}$

Excel:

$p(x)=P(X=x)=$ HYPGEOM.DIST $(x ; n ; M ; N ; 0)$

$F(x)=P(X \leq x)=$ HYPGEOM.DIST $(x ; n ; M ; N ; 1)$

Example 20

A box contains 150 good light bulbs and 50 defective bulbs. We randomly choose 30 bulbs. What is the probability that at least 25 bulbs are good?

Let $X$ denote the number of good bulbs of selected 30 bulbs. Then the probability distribution of $X$ is hypergeometric with parameters $n=30, N=200, M=150$.

The probability that at least 25 bulbs are good is 


$$
P(X \geq 25)=1-P(X \leq 24)=1-\sum_{x=0}^{24} \frac{\left(\begin{array}{c}
150 \\
x
\end{array}\right)\left(\begin{array}{c}
50 \\
30-x
\end{array}\right)}{\left(\begin{array}{c}
200 \\
30
\end{array}\right)}
$$

Excel:

$$
P(X \geq 25)=1-P(X \leq 24)=1-\text { HYPGEOM.DIST }(24 ; 30 ; 150 ; 200 ; 1) \doteq 0.18
$$

Poisson distribution $P(\lambda)$

$$
p(x)=\frac{\lambda^{x}}{x !} \mathrm{e}^{-\lambda}, \quad x=0,1, \ldots
$$

where $\lambda$ is the average number of successes in the given time interval or region of space.

$E(X)=D(X)=\lambda$

Excel:

$p(x)=P(X=x)=\operatorname{POISSON.DIST}(x ; \lambda ; 0)$

$F(x)=P(X \leq x)=$ POISSON.DIST $(x ; \lambda ; 1)$

If $X$ counts the number of events in the interval $[0,1]$ and $\lambda$ is the average number that occur in unit time, then $X \sim P(\lambda t)$, that is,

$$
p(x)=\frac{(\lambda t)^{x}}{x !} \mathrm{e}^{-\lambda t}, \quad x=0,1, \ldots
$$

If binomial distribution $n \rightarrow \infty, p \rightarrow 0$ such that $n p=\lambda$ then binomial distribution tends to Poisson distribution.

\section{Example 21}

A book contains 300 pages. The mean number of typing errors in a book is 1.5 per page. Find the probability that on a page chosen at random there are 0 mistakes.

Let $X$ denote the number of mistakes on the page. Then the probability distribution of $X$ is Poisson with parameter $\lambda=1.5$.

The probability that on the page are no mistakes is

$$
P(X=0)=\frac{1.5^{0}}{0 !} \mathrm{e}^{-1.5} \doteq 0.223
$$

Excel:

$$
P(X=0)=\text { POISSON.DIST }(0 ; 1.5 ; 0) \doteq 0.223
$$

Exercise 22

a) A company is considering drilling four oil wells. The probability of success for each well is 0.40 , independent of the results for any other well. What is the probability that one or more wells will be successful? What is the expected number of successes? 
b) Customers arrive at a checkout counter at an average rate of 1.5 per minute. Find the probabilities that at least three will arrive during an interval of two minutes?

c) Twelve doughnuts sampled from a manufacturing process are weighed each day. The probability that a sample will have no doughnuts weighing less than the design weight is $6.872 \%$. What is the probability that a sample of twelve doughnuts contains exactly three doughnuts weighing less than the design weight?

d) The number of meteors found by a radar system in any 30-second interval under specified conditions averages 1.81. Assume the meteors appear randomly and independently. What is the probability of observing at least five but not more than eight meteors in two minutes of observation?

e) A telephone number is selected at random from a directory. Let $X$ denote the last digit of randomly selected telephone number. Find the probability that the last digit of the selected number is greater than or equal to 7 . 


\section{Continuous probability distribution}

Uniform distribution $U(a, b)$

$$
f(x)=\left\{\begin{array}{ll}
\frac{1}{b-a} & x \in[a, b] \\
0 & x \notin[a, b]
\end{array} .\right.
$$

$E(X)=\frac{a+b}{2}, D(X)=\frac{(b-a)^{2}}{12}$

\section{Example 23}

The amount of time that a person must wait for a bus is uniformly distributed between zero and 20 minutes, inclusive. What is the probability that a person waits fewer than 12 minutes? Find the 95 th percentile.

Let $X$ denote the number of minutes (the waiting time at a bus stop). The waiting time is between 0 and 20 minutes $\Rightarrow X \sim U(0,20)$.

The probability that a person waits less than 12 minutes is

$$
P(X<12)=F(12)=\frac{12-0}{20-0}=0.6
$$

Let $x=95$ th percentile $\Rightarrow P(X<x)=0.95$

$$
\begin{aligned}
P(X<x) & =\frac{x-0}{20-0} \\
0.95 & =\frac{x}{20} \\
x & =0.95 \cdot 20=19
\end{aligned}
$$

Ninety-five percent of the time, a person must wait at most 19 minutes.

Exponential distribution $\operatorname{Exp}(\lambda)$

$$
f(x)=\left\{\begin{array}{ll}
\lambda \mathrm{e}^{-\lambda x} & x \in[0,+\infty) \\
0 & x \in(-\infty, 0)
\end{array},\right.
$$

where $\lambda$ is the intensity or the rate at which an event occurs.

$E(X)=\frac{1}{\lambda}, D(X)=\frac{1}{\lambda^{2}}$

For $x>0$ the cumulative distribution function is

$$
F(x)=1-\mathrm{e}^{-\lambda x}
$$


Excel:

$f(x)=\operatorname{EXPON} . D I S T(x ; \lambda ; 0)$

$F(x)=P(X \leq x)=\operatorname{EXPON} . \operatorname{DIST}(x ; \lambda ; 1)$

A continuous memoryless distribution that describes the time between events in a Poisson process

$$
P\left(X>x+x_{0} \mid X>x_{0}\right)=P(X>x) .
$$

Is related to the Poisson distribution, although the exponential distribution is continuous whereas the Poisson distribution is discrete.

\section{Example 24}

The time (in hours) required to repair a machine is an exponentially distributed random variable with parameter $\lambda=1 / 3$. Find

a) the probability that a repair time takes between 1 and 3 hours.

b) the conditional probability that a repair time takes at least 7 hours, given that its duration exceeds 5 hours.

Let $X$ denote the time required to repair a machine. The average duration of repair is 3 hours $\Rightarrow X \sim \operatorname{Exp}(1 / 3)$.

a)

The probability that a repair time takes between 1 and 3 hours is

$$
P(1 \leq X \leq 3)=F(3)-F(1)=\left(1-\mathrm{e}^{-3 / 3}\right)-\left(1-\mathrm{e}^{-1 / 3}\right)=\mathrm{e}^{-1 / 3}-\mathrm{e}^{-1} \doteq 0.35
$$

Excel: $P(1 \leq X \leq 3)=$ EXPON.DIST $(3 ; 1 / 3 ; 1)-$ EXPON.DIST $(1 ; 1 / 3 ; 1) \doteq 0.35$

b)

The conditional probability that a repair time takes at least 7 hours, given that its duration exceeds 5 hours is

$$
\begin{aligned}
P(X \geq 7 \mid X>5) & =\frac{P(X \geq 7)}{P(X>5)} \\
& =\frac{1-P(X<7)}{1-P(X \leq 5)} \\
& =\frac{1-\left(1-\mathrm{e}^{-7 / 3}\right)}{1-\left(1-\mathrm{e}^{-5 / 3}\right)} \\
& \doteq 0.51
\end{aligned}
$$

The second way how to calculate this problem is using memoryless property of exponential distribution,

$$
\begin{aligned}
P(X>2+5 \mid X>5) & =P(X>2) \\
& =1-P(X \leq 2) \\
& =\mathrm{e}^{-2 / 3} \\
& \doteq 0.51
\end{aligned}
$$


Normal distribution $\mathrm{N}\left(\mu, \sigma^{2}\right)$

$$
f(x)=\frac{1}{\sigma \sqrt{2 \pi}} \mathrm{e}^{-\frac{1}{2}\left(\frac{x-\mu}{\sigma}\right)^{2}}, \quad x \in(-\infty,+\infty),
$$

where $\mu$ is called the location parameter (as it changes the location of density curve) and $\sigma^{2}$ is called the scale parameter of normal distribution (as it changes the scale of density curve).

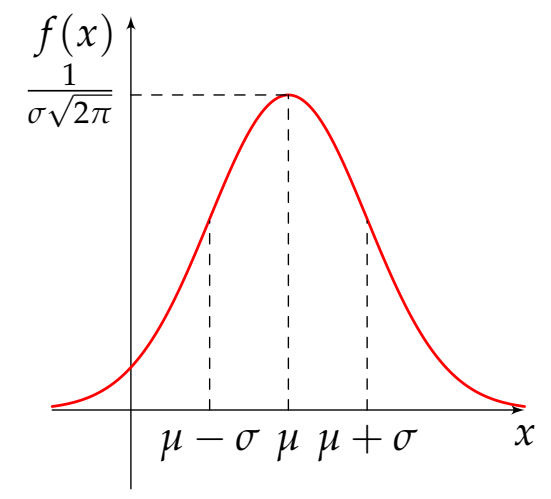

$E(X)=\mu, D(X)=\sigma^{2}, A=0, \bar{e}=0$

Excel:

$f(x)=\operatorname{NORM}$ DIST $(x ; \mu ; \sigma ; 0)$

$F(x)=\operatorname{NORM} \cdot \operatorname{DIST}(x ; \mu ; \sigma ; 1)$

$x_{p}=\operatorname{NORM} . \mathrm{INV}(p ; \mu ; \sigma)$

Example 25

A factory produces lamps, the lifetimes of which follow the normal distribution. The average lifetime of a lamp is 800 hours with a standard deviation of 40 hours.

a) What percentage of lamps will last between 750 and 800 hours?

b) After how many burning hours would we expect $5 \%$ of the lamps to be left?

Let $X$ denote the lamps lifetime $\Rightarrow X \sim N\left(800,40^{2}\right)$.

a) What percentage of lamps will last between 750 and 800 hours?

$$
P(750 \leq X \leq 800)=\text { NORM.DIST }(800 ; 800 ; 40 ; 1)-\operatorname{NORM} . D I S T(750 ; 800 ; 40 ; 1) \doteq 0.39
$$

b) After how many burning hours would we expect $5 \%$ of the lamps to be left? This corresponds to the time at which

$$
\begin{gathered}
P(X>x)=0.05 \\
P(X<x)=1-0.05=0.95
\end{gathered}
$$

We need calculate $95 \%$ quantile of the random variable $X$.

$$
x_{0.95}=\operatorname{NORM} . I N V(0.95 ; 800 ; 40) \doteq 866
$$

Then after 866 hours of burning, we would expect $5 \%$ of the lamps to be left. 


\section{Standard normal distribution $\mathrm{N}(0,1)$}

We can transform all the observations of any normal random variable $X$ with mean $\mu$ and variance $\sigma^{2}$ to a new set of observations of another normal random variable $Z$ with $\mu=0$ and $\sigma^{2}=1$ using the following transformation:

$$
\mathrm{Z}=\frac{X-\mu}{\sigma}
$$

Distribution function od standard normal distribution $\Phi(z)$ is usually tabulated only for positive values of $z$ due to the symmetry of $\Phi$ around zero:

$$
\Phi(-z)=P(Z \leq-z)=P(Z>z)=1-P(Z \leq z)=1-\Phi(z)
$$

Excel: $\varphi(x)=$ NORM.S.DIST $(x ; 0)$

$\Phi(x)=\operatorname{NORM} . S . D I S T(x ; 1)$

$x_{p}=\operatorname{NORM} . S . I N V(p)$

Example 26

Let $X$ be the score on the IQ test. The score follow the normal distribution with a mean of 100 and a standard deviation of 15 .

a) What is $P(95 \leq X \leq 115)$ ?

b) What is the lowest possible IQ score that a person can have and still be in the top $1 \%$ of all IQ scores?

a) We express the distribution function of $X$ in terms of the distribution function of a standard normal random variable $Z$.

$$
\begin{aligned}
P(95 \leq X \leq 115) & =P(X \leq 115)-P(X \leq 95) \\
& =P\left(Z \leq \frac{115-100}{15}\right)-P\left(Z \leq \frac{95-100}{15}\right) \\
& =P(Z \leq 1)-P(Z \leq-0.33) \\
& =\Phi(1)-(1-\Phi(0.33)) \\
& =0.8413-(1-0.6293) \doteq 0.47
\end{aligned}
$$

Excel:

$$
\begin{aligned}
& P(-0.33 \leq Z \leq 1)=\text { NORM.S.DIST }(1 ; 1)-\operatorname{NORM.S.DIST}(-0.33 ; 1) \doteq 0.47 \\
& P(95 \leq X \leq 115)=\text { NORM.DIST }(115 ; 100 ; 15 ; 1)-\operatorname{NORM} . D I S T(95 ; 100 ; 85 ; 1) \doteq 0.47
\end{aligned}
$$

b) If a person is in the top $1 \%$, then that means that $99 \%$ of the people have lower IQ scores $P(X<x)=0.99$. So

$$
P(X<x)=P\left(Z<\frac{x-100}{15}\right)=\Phi\left(\frac{x-100}{15}\right)=0.99
$$

From table

$$
0.99 \approx \Phi(2.33) \Rightarrow \frac{x-100}{15}=2.33 \Rightarrow x=135
$$

Excel:

$$
\begin{aligned}
& x_{0.99}=15 \cdot \operatorname{NORM} . S . I N V(0.99)+100 \doteq 135 \\
& x_{0.99}=\operatorname{NORM} . \operatorname{INV}(0.99 ; 100 ; 15) \doteq 135
\end{aligned}
$$

A person with IQ score 135 or higher falls in the top $1 \%$ of all IQ scores. 


\subsection{Normal approximation}

\section{Binomial approximation}

Let $X$ be a binomial random variable with number of trials $n$ and probability of success $p$. In situation, when $n$ is large and $p$ is close to 0.5 , we can use the normal distribution with $\mu=n p$ and $\sigma^{2}=n p(1-p)$.

That is

$$
X \sim \operatorname{Bi}(n, p) \rightarrow X \sim \mathrm{N}(n p, n p(1-p))
$$

The general conditions for using normal approximation to binomial distribution are $n p \geq 5$ and $n(1-p) \geq 5$.

\section{Poisson approximation}

Let $X$ be a Poisson random variable with mean $\lambda$. For large value of the $\lambda$ we can use the normal distribution with $\mu=\lambda$ and $\sigma^{2}=\lambda$.

That is

$$
X \sim P(\lambda) \rightarrow X \sim \mathrm{N}(\lambda, \lambda)
$$

The general condition for using normal approximation to Poisson distribution is $\lambda \geq 5$.

\section{Continuity correction}

The binomial and Poisson distributions are discrete random variables, whereas the normal distribution is continuous. We are approximating a discrete distribution with a continuous one, and so we need to make a continuity correction.

$$
\begin{aligned}
P(X=a) & =P(a-0.5<X<a+0.5) \\
P(X<a) & =P(X<a-0.5) \\
P(X \leq a) & =P(X<a+0.5) \\
P(a<X \leq b) & =P(a-0.5<X<b+0.5) \\
P(a \leq X<b) & =P(a-0.5<X<b-0.5)
\end{aligned}
$$

\section{Example 27}

Consider tossing a coin 35 times. What is the probability of getting between 11 and 14 heads?

Let $X$ denote the number of heads thrown $\Rightarrow X \sim B i\left(35 ; \frac{1}{2}\right)$.

Since $p$ equals 0.5 , we use normal approximation to binomial distribution.

In our example, $n p=35 \cdot 0.5=17.5$ and $n(1-p)=35 \cdot(1-0.5)=17.5$.

$n p \geq 5, n(1-p) \geq 5$ and $p=0.5$

$\Rightarrow$ we can use the normal approximation $X \sim N(35 \cdot 0.5 ; 35 \cdot 0.5 \cdot(1-0.5))$, so $X \sim N(17.5 ; 8.75)$. 


$$
\begin{aligned}
P(11 \leq X \leq 14) & =P(11-0.5<X<14+0.5) \\
& =P(10.5<X<14.5) \\
& =F(14.5)-F(10.5) \\
& =\operatorname{NORM.DIST}(14.5 ; 17.5 ; \sqrt{8.75} ; 1)-\operatorname{NORM.DIST}(10.5 ; 17.5 ; \sqrt{8.75} ; 1) \\
& \doteq 0.146
\end{aligned}
$$

Exercise 28

a) Diameters of bolts produced by a particular machine are normally distributed with mean $0.760 \mathrm{~cm}$ and standard deviation $0.012 \mathrm{~cm}$. Specifications call for diameters from $0.720 \mathrm{~cm}$ to $0.780 \mathrm{~cm}$. What percentage of bolts will meet these specifications? What percentage of bolts will be smaller than $0.730 \mathrm{~cm}$ ?

b) The number of days ahead travelers purchase their airline tickets can be modeled by an exponential distribution with the average amount of time equal to 15 days. Find the probability that a traveler will purchase a ticket fewer than ten days in advance. How many days do half of all travelers wait?

c) A subway train on the Red Line arrives every eight minutes during rush hour. We are interested in the length of time a commuter must wait for a train to arrive. The time follows a uniform distribution. Find the probability that the commuter waits less than one minute. Find the probability that the commuter waits between three and four minutes.

d) Suppose you are testing a new software, and a bug causes errors randomly at a constant rate of three times per hour. What is the probability that the first bug will occur within the first ten minutes?

e) The average life of a certain type of motor is 12 years, with a standard deviation of 3 years. If the manufacturer is willing to replace only $4 \%$ of the motors because of failures, how long a guarantee should he offer? Assume that the lives of the motors follow a normal distribution.

f) The average number of collisions occurring in a year at a particular intersection is 54. Assume that the requirements of the Poisson distribution are satisfied. What is the probability of exactly 40 collisions in a year? 


\section{Descriptive statistics: summary numbers}

\subsection{Measures of location}

Measures of location - describe the central tendency of the data. They include means, mode, median, and quantiles. Consider the data set of $N$ measurements $x_{1}, x_{2}, \ldots, x_{N}$, which represents the complete population or the sample taken from the population.

\section{Means (averages)}

\section{Arithmetic mean}

Population mean:

$$
\mu=\frac{1}{N} \sum_{i=1}^{N} x_{i}
$$

Sample mean:

$$
\bar{x}=\frac{1}{N} \sum_{i=1}^{N} x_{i}
$$

\section{Geometric mean}

$$
G\left(x_{1}, x_{2}, \ldots, x_{N}\right)=\sqrt[N]{x_{1} \cdot x_{2} \cdot \ldots \cdot x_{N}}
$$

\section{Harmonic mean}

$$
\overline{x_{h}}=\frac{N}{\frac{1}{x_{1}}+\frac{1}{x_{2}}+\cdots+\frac{1}{x_{N}}}
$$

2. Mode $(M o, \hat{x})$

- the value that appears most frequently or the midpoint of the class with the largest frequency (in case of grouped frequency approach)

3. Median $(M e, \tilde{x})$

- the middle item in a sample of values ordered from the smallest to the largest (in case the number of items is odd) or the arithmetic mean of the two middle items (in case the number of items is even)

\section{Quantiles (quartiles, deciles, percentiles) $\left(Q(p), x_{p}\right)$}

- quantiles are the cutpoints dividing a set of observations (ordered from the smallest to the largest) into equal sized groups (for example quartiles divide the range of values into four parts, each containing one quarter of the values)

- quantile $Q(p)$ is the $i$-th object in the ordered sample where $i=N \cdot p+0.5$ (in case $i$ is integer) or the arithmetic mean of the two adjacent items (in case $i$ is not integer)

- lower quartile $x_{0.25}$ means that about $25 \%$ of the numbers in the data set lie below $x_{0.25}$ and about $75 \%$ lie above $x_{0.25}$

- upper quartile $x_{0.75}$ means that about $75 \%$ of the numbers in the data set lie below $x_{0.75}$ and about $25 \%$ lie above $x_{0.75}$ 


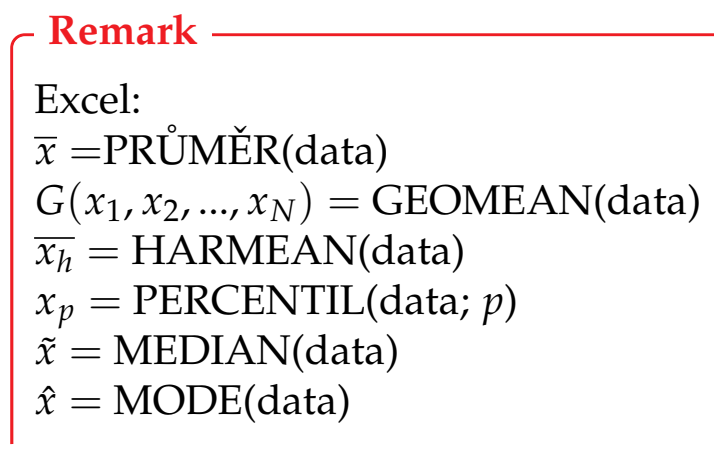

\subsection{Measures of variability}

Measures of variability - describe the spread of the data. They include sample range, interquartile range, mean absolute deviation from the mean, variance, standard deviation and coefficient of variation.

1. Sample range $(R)$

- the difference between the largest item $x_{\max }$ and the smallest item $x_{\min }$ in the data sample

$$
R=x_{\max }-x_{\min }
$$

2. Interquartile range $(I Q R)$

- the difference between the upper quartile and the lower quartile

$$
I Q R=x_{0.75}-x_{0.25}
$$

3. Mean absolute deviation from the mean $(M A D)$

Population mean absolute deviation from the mean:

$$
M A D=\frac{1}{N} \sum_{i=1}^{N}\left|x_{i}-\mu\right|
$$

Sample mean absolute deviation from the mean:

$$
\widehat{M A D}=\frac{1}{N} \sum_{i=1}^{N}\left|x_{i}-\bar{x}\right|
$$

\section{Variance}

- the variance has units of the quantity squared, for example $m^{2}$ or $s^{2}$ if the original quantity was measured in meters or seconds, respectively

Population variance:

$$
\sigma^{2}=\frac{1}{N} \sum_{i=1}^{N}\left(x_{i}-\mu\right)^{2}
$$

Sample variance:

$$
s^{2}=\frac{1}{N-1} \sum_{i=1}^{N}\left(x_{i}-\bar{x}\right)^{2}
$$

5. Standard deviation $(\sigma, s)$

- the square root of the variance 
- the standard deviation has the same units as the original data

\section{Coefficient of variation}

- the ratio between the standard deviation and the mean for the same set of data, expressed as a percentage

Population coefficient of variation:

$$
c_{v}=\frac{\sigma}{\mu}
$$

Sample coefficient of variation:

$$
\widehat{c}_{v}=\frac{s}{\bar{x}}
$$

\section{Example 29}

Consider the sample consisting of the following nine results: 2.3, 7.2, 3.7, 4.6, 5.0, 7.0, 3.7, 4.9, 4.2. Take the given data as a sample and calculate: mean, mode, quartiles, sample range, interquartile range, mean absolute deviation from the mean, variance, standard deviation, coefficient of variation.

Sample mean:

$$
\bar{x}=\frac{1}{N} \sum_{i=1}^{N} x_{i}=\frac{1}{9}(2.3+7.2+3.7+4.6+5.0+7.0+3.7+4.9+4.2) \doteq 4.733
$$

Mode:

$$
M o=3.7
$$

Quartiles:

- the first step to find quartiles is to sort the data in order of increasing magnitude, giving the following sequence:

$$
2.3,3.7,3.7,4.2,4.6,4.9,5.0,7.0,7.2
$$

- the second step is to use the formula $i=N \cdot p+0.5$ and find the $i$-th object in the ordered sample, which equals $Q(p)$

1. The first quartile (the lower quartile) $Q(0.25)$ :

$$
i=N \cdot p+0.5=9 \cdot 0.25+0.5=2.75,
$$

so we need to find the objects with the order numbers 2 and 3 and make their arithmetic mean

$$
Q(0.25)=\frac{3.7+3.7}{2}=3.7
$$

2. The second quartile (the median) $Q(0.5)$ :

$$
i=N \cdot p+0.5=9 \cdot 0.5+0.5=5,
$$

so we need to find the object with the order number 5

$$
Q(0.5)=4.6
$$


3. The third quartile (the upper quartile) $Q(0.75)$ :

$$
i=N \cdot p+0.5=9 \cdot 0.75+0.5=7.25,
$$

so we need to find the objects with the order numbers 7 and 8 and make their arithmetic mean

$$
Q(0.75)=\frac{5+7}{2}=6
$$

Sample range:

$$
R=x_{\max }-x_{\min }=7.2-2.3=4.9
$$

Interquartile range:

$$
I Q R=Q(0.75)-Q(0.5)=6-3.7=2.3
$$

Mean absolute deviation from the mean:

$$
\widehat{M A D}=\frac{1}{N} \sum_{i=1}^{N}\left|x_{i}-\bar{x}\right| \doteq \frac{1}{9}(|2.3-4.733|+|3.7-4.733|+\ldots+|7.2-4.733|) \doteq 1.148
$$

Sample coefficient of variation:

$$
\widehat{c}_{v}=\frac{s}{\bar{x}} \doteq \frac{1.568}{4.733} \doteq 0.331
$$

Sample variance:

$s^{2}=\frac{1}{N-1} \sum_{i=1}^{N}\left(x_{i}-\bar{x}\right)^{2} \doteq \frac{1}{8}\left((2.3-4.733)^{2}+(3.7-4.733)^{2}+\ldots+(7.2-4.733)^{2}\right) \doteq 2.460$

Sample standard deviation:

$$
s=\sqrt{s^{2}} \doteq \sqrt{2.460} \doteq 1.568
$$

\section{Exercise 30}

a) The same dimension was measured on each of six successive parts as they came off a production line. The results were $21.14 \mathrm{~mm}, 21.87 \mathrm{~mm}, 21.53 \mathrm{~mm}, 21.37 \mathrm{~mm}$, $21.61 \mathrm{~mm}$ and $21.93 \mathrm{~mm}$. Calculate the mean, median, variance, standard deviation and coefficient of variation.

i) Consider this set of values as a complete population.

ii) Consider this set of values as a sample of all possible measurements.

b) Four items in a sequence were measured as 50, 160, 100, and $400 \mathrm{~mm}$. Find their arithmetic mean, geometric mean, harmonic mean and median.

c) The times to perform a particular step in a production process were measured repeatedly. The times were 20.3 s, 19.2 s, 21.5 s, 20.7 s, 22.1 s, 19.9 s, 21.2 s, 20.6 s. Calculate the arithmetic mean, geometric mean, median, lower quartile, upper quartile, variance, standard deviation and coefficient of variation, considering this set of values as a sample of all possible measurements of the times for this step in the process. 


\section{Grouped frequencies and graphical descriptions}

\subsection{Stem-and-leaf display}

\section{Example 31}

Data have been obtained on the lives of batteries of a particular type in an industrial application. The following numbers represents the lives of 36 batteries recorded to the nearest tenth of a year:

$4.1,5.2,2.8,4.9,5.6,4.0,4.1,4.3,5.4,4.5,6.1,3.7,2.3,4.5,4.9,5.6,4.3,3.9,3.2,5.0,4.8,3.7$, $4.6,5.5,1.8,5.1,4.2,6.3,3.3,5.8,4.4,4.8,3.0,4.3,4.7,5.1$.

Make a stem-and-leaf display for these data. Show the leaves sorted in order of increasing magnitude on each stem.

For these data we choose the digits before the decimal point $(1,2,3,4,5,6)$ as the stems and we put the digits after the decimal point as the leaves on its corresponding stem.

- the decimal point is not usually shown

- the leaves are often sorted in order of increasing magnitude on each stem

- the number of stems on each leaf can be counted and shown as a frequency

$\begin{array}{lll}\text { Stem } & \text { Leaf } & \text { Frequency } \\ 1 & 8 & 1 \\ 2 & 38 & 2 \\ 3 & 023779 & 6 \\ 4 & 0112333455678899 & 16 \\ 5 & 011245668 & 9 \\ 6 & 13 & 2\end{array}$

\subsection{Box plot (box-and-whisker plot)}

Box plot is a narrow box extends from the lower quartile to the upper quartile, where the median is marked by a line extending across the box. The smallest and the largest value are marked, and each is joined to the box by a straight line, the whisker.

Example 32

Make a box plot for the data from previous example.

The first step to make a box plot is to sort the data in order of increasing magnitude and find the minimum, maximum, lower quartile, upper quartile and median. The sorted data: $1.8,2.3,2.8,3.0,3.2,3.3,3.7,3.7,3.9,4.0,4.1,4.1,4.2,4.3,4.3,4.3,4.4,4.5,4.5,4.6,4.7,4.8,4.8$, $4.9,4.9,5.0,5.1,5.1,5.2,5.4,5.5,5.6,5.6,5.8,6.1,6.3$.

- minimum: $x_{\min }=1.8$

- maximum: $x_{\max }=6.3$

- lower quartile: $Q(0.25)=3.95$

- median: $M e=Q(0.5)=4.5$

- upper quartile: $Q(0.75)=5.1$ 


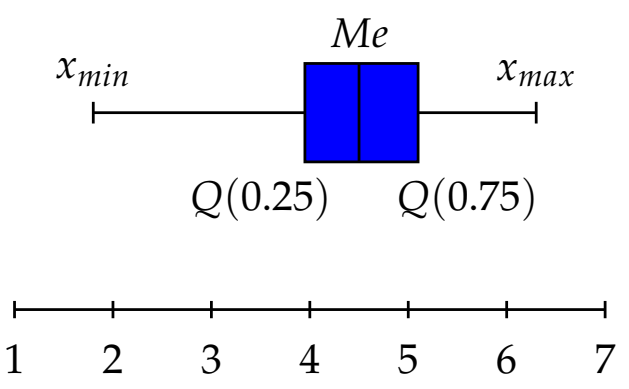

\subsection{Bar chart (bar graph)}

Bar chart presents discrete data with rectangular bars with heights proportional to the values that they represent. One axis of the chart shows the specific categories being compared and the other axis represents a measured value.

\section{Example 33}

The numbers of defective items in successive samples of six items were detected and summarized in the frequency table below. Make a bar graph for the data.

$\begin{array}{cc}\text { Number of defectives } x_{i} & \text { Frequency } f_{i} \\ 0 & 4 \\ 1 & 10 \\ 2 & 6 \\ 3 & 5 \\ 4 & 2\end{array}$

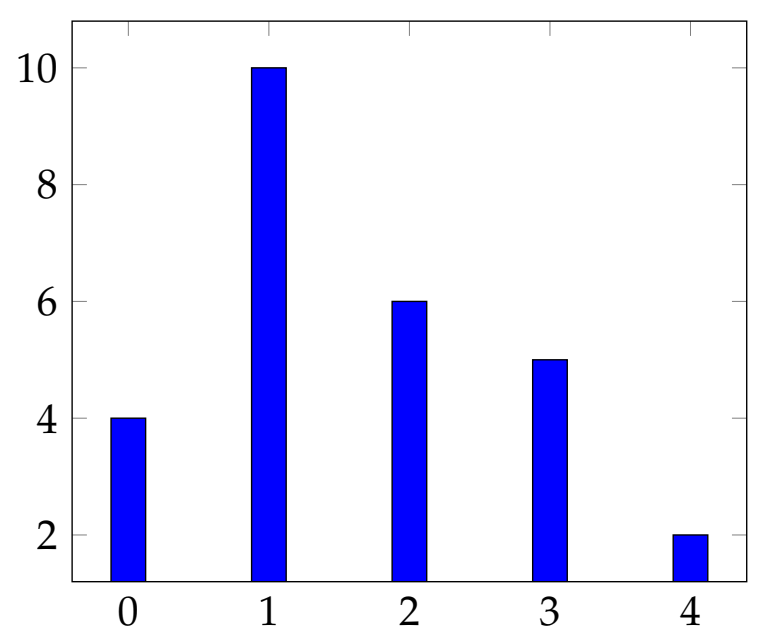

\subsection{Graphs of continuous data}

The continuous data are divided into intervals (classes) and the frequency of occurrence for each class is counted to make the data easier to comprehend (the grouped frequency approach). The appropriate number of classes is given by Sturges' Rule: number of class intervals $\approx 1+3.3 \log N$ (where $N$ is the total number of observations in the sample or population). The class boundaries must be clear with no gaps and no overlaps (e.g. if the values are stated to two decimal places, the class boundaries should end in five in the third decimal). 


\section{Example 34}

Over a period of 60 days the percentage relative humidity in a storage building was measured. Mean daily values were recorded as shown below. Make a graph of the data. $60,63,64,71,67,73,79,80,83,81,86,90,96,98,98,99,89,80,77,78,71,79,74,84,85,82$, $90,78,79,79,78,80,82,83,86,81,80,76,66,74,81,86,84,72,79,72,84,79,76,79,74,66$, $84,78,91,81,64,76,78,82$

The total number of observations: $N=60$

The appropriate number of classes: $\approx 1+3.3 \log N \doteq 6.868$

The class width: $\approx\left(x_{\max }-x_{\min }\right) / 6.686=(99-60) / 6.686 \doteq 5.68$

Thus we can make a frequency table with 7 classes of the width equal to 6 .

$\begin{array}{ccccccc}\begin{array}{c}\text { Lower class } \\ \text { boundary }\end{array} & \begin{array}{c}\text { Upper class } \\ \text { boundary }\end{array} & \begin{array}{c}\text { Class } \\ \text { midpoint }\end{array} & \begin{array}{c}\text { Class } \\ \text { freq. }\end{array} & \begin{array}{c}\text { Cumulative } \\ \text { freq. }\end{array} & \begin{array}{c}\text { Relative } \\ \text { freq. }\end{array} & \begin{array}{c}\text { Cumulative } \\ \text { relative freq. }\end{array} \\ 68.5 & 64.5 & 61.5 & 4 & 4 & 0.067 & 0.067 \\ 74.5 & 70.5 & 67.5 & 3 & 7 & 0.05 & 0.117 \\ 76.5 & 76.5 & 73.5 & 11 & 18 & 0.183 & 0.3 \\ 82.5 & 82.5 & 79.5 & 24 & 42 & 0.4 & 0.7 \\ 88.5 & 88.5 & 85.5 & 10 & 52 & 0.167 & 0.867 \\ 94.5 & 94.5 & 91.5 & 4 & 56 & 0.067 & 0.933 \\ & 100.5 & 97.5 & 4 & 60 & 0.067 & 1\end{array}$

- class midpoint: the point halfway between the corresponding class boundaries

- class frequency: the number of items in the class

- cumulative freq.: the total of all class frequencies smaller than a class boundary

- relative freq.: the class frequency divided by the total number of observations

- relative cumulative freq.: the total of all relative frequencies smaller than a class boundary

\subsection{Histogram}

Histogram is the bar graph in which the class frequency or relative class frequency is plotted against values of the quantity being studied (so the height of the bar indicates the class frequency or relative class frequency). The class midpoints are plotted along the horizontal axis. Histogram for continuous data should have the bars touching one another.

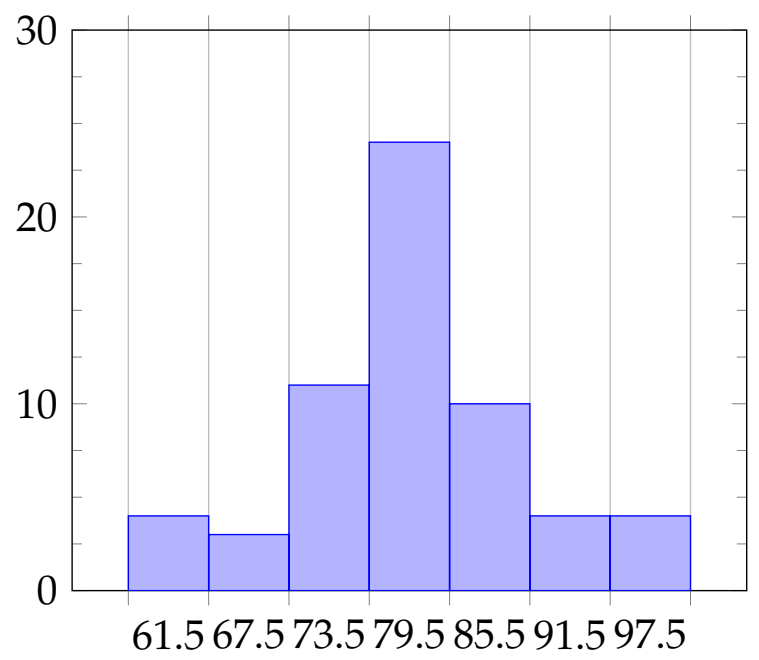




\subsection{Cumulative frequency diagram}

Cumulative frequency diagram is a plot of cumulative frequency vs. the upper class boundary with successive points joined by straight lines. It could be changed into a relative cumulative frequency diagram by a change of scale for the ordinate.

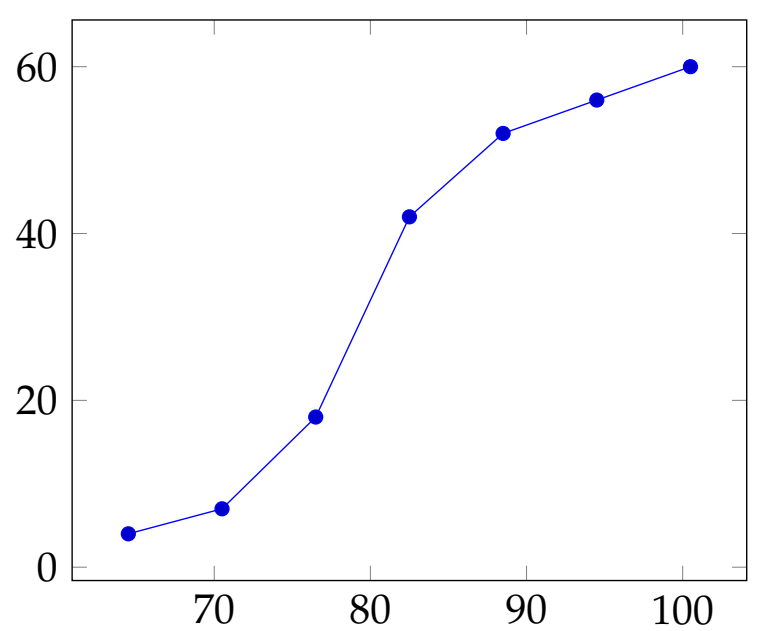

\section{Exercise 35}

A random sample was taken of the thickness of insulation in transformer windings, and the following thicknesses (in millimeters) were recorded:

$18,21,22,29,25,31,37,38,41,39,44,48,54,56,56,57,47,38,35,36,29,37,32,42,43,40$, $48,36,37,37,36,38,40,41,44,39,38,34,24,32,39,44,42,30,37,30,42,37,34,37,32,24$, $42,36,49,39,23,34,36,40$.

a) Make a stem-and-leaf display for these data.

b) Find the mode, median, lower quartile, and ninth decile of these data.

c) Make a frequency table for the data. Use Sturges' rule.

d) Draw a frequency histogram, a cumulative frequency graph and box plot. 


\section{Sampling and combination of variables}

\subsection{Linear combination of independent variables}

\section{Properties of mean (expected value)}

1. $\mu(c)=c$

2. $\mu(c X)=c \cdot \mu(X)$

3. $\mu(X+Y)=\mu(X)+\mu(Y)$

4. $\mu(X \cdot Y)=\mu(X) \cdot \mu(Y)$
Properties of variance

1. $\sigma^{2}(c)=0$

2. $\sigma^{2}(c X)=c^{2} \cdot \sigma^{2}(X)$

3. $\sigma^{2}(a X+b)=a^{2} \cdot \sigma^{2}(X)$

4. $\sigma^{2}(X+Y)=\sigma^{2}(X)+\sigma^{2}(Y)$

\section{Example 36}

Cans of corn have a mean content of $300 \mathrm{~g}$ with a standard deviation of $6 \mathrm{~g}$. There are 24 cans in a case. What is the mean and the standard deviation of the content of a case?

$X_{i} \ldots$ content of $i$-th can, $i=1, \ldots, 24$

$Y \ldots$ content of one case ( 24 cans), $Y=X_{1}+\cdots+X_{24}$

We have:

$\mu\left(X_{i}\right)=300, i=1, \ldots, 24$

$\sigma\left(X_{i}\right)=6, i=1, \ldots, 24$

$\sigma^{2}\left(X_{i}\right)=6^{2}=36, i=1, \ldots, 24$

Variables $X_{1}, \ldots, X_{24}$ are independent, so:

$$
\begin{gathered}
\mu(Y)=\mu\left(X_{1}\right)+\cdots+\mu\left(X_{24}\right)=300+\cdots+300=24 \cdot 300=7200 \\
\sigma^{2}(Y)=\sigma^{2}\left(X_{1}\right)+\cdots+\sigma^{2}\left(X_{24}\right)=36+\cdots+36=24 \cdot 36=864 \\
\sigma(Y)=\sqrt{\sigma^{2}(Y)}=\sqrt{864} \doteq 29.4
\end{gathered}
$$

The mean content of a case is $7200 \mathrm{~g}$, the standard deviation is $29.4 \mathrm{~g}$.

\section{Example 37}

The circumference of a board with rectangular cross-section is twice the sum of the width and thickness of the board. Knowing the variance of the width and the variance of the thickness, what is the variance of the circumference?

$X \ldots$ width of a board

$Y$...thickness of a board

$Z$... circumference of a board

We have:

$$
\begin{gathered}
Z=2(X+Y) \\
\sigma^{2}(Z)=2^{2}\left(\sigma^{2}(X)+\sigma^{2}(Y)\right)
\end{gathered}
$$




\subsection{Sampling}

A population is the entire group of objects or possible measurements in which we are interested. A sample is a group of objects or readings taken from a population.

\section{Sample mean}

$$
\bar{X}=\frac{X_{1}+X_{2}+\cdots+X_{n}}{n}
$$

$X_{1}, \ldots, X_{n} \ldots$ random variables with the same mean $\mu$ and the same variance $\sigma^{2}$ $n$... sample size

Characteristics of sample mean: $\mu(\bar{X}), \sigma^{2}(\bar{X}), \sigma(\bar{X})$

1. Sampling with replacement $\left(X_{1}, \ldots, X_{n} \ldots\right.$ independent random variables)

- $\mu(\bar{X})=\mu$

- $\sigma^{2}(\bar{X})=\frac{\sigma^{2}}{n}$

- $\sigma(\bar{X})=\frac{\sigma}{\sqrt{n}}$

2. Sampling without replacement $\left(X_{1}, \ldots, X_{n} \ldots\right.$ dependent random variables)

- $\mu(\bar{X})=\mu$

- $\sigma^{2}(\bar{X})=\frac{\sigma^{2}}{n} \cdot \frac{N-n}{N-1}$

- $\sigma(\bar{X})=\frac{\sigma}{\sqrt{n}} \cdot \frac{\sqrt{N-n}}{\sqrt{N-1}}$

$N$...population size

\section{Example 38}

A population of size 20 is sampled without replacement. The standard deviation of the population is 0.35 . We require the standard error of the mean to be no more than 0.15 . What is the minimum sample size?

In case of sampling without replacement we use the formula:

$$
\sigma(\bar{X})=\frac{\sigma}{\sqrt{n}} \cdot \frac{\sqrt{N-n}}{\sqrt{N-1}}
$$

In this case we have $\sigma=0.35, N=20$, and substitute the limiting value of 0.15 for $\sigma(\bar{X})$ :

$$
\begin{gathered}
\frac{0.35}{\sqrt{n}} \cdot \frac{\sqrt{20-n}}{\sqrt{20-1}} \leq 0.15 \\
20-n \leq 3.490 n
\end{gathered}
$$




$$
\begin{gathered}
n \geq \frac{20}{4.490} \doteq 4.45 \\
n=5
\end{gathered}
$$

As the sample size $n$ must be an integer, the minimum sample size is 5 .

\section{Example 39}

The standard deviation of measurements of a linear dimension of a mechanical part is $0.14 \mathrm{~mm}$. What sample size is required if the standard error of the mean must be no more than $0.04 \mathrm{~mm}$ ?

Since the dimension can be measured as many times as desired, the population size $N$ is effectively infinite and we use the formula for sampling with replacement:

$$
\sigma(\bar{X})=\frac{\sigma}{\sqrt{n}}
$$

In this case we have $\sigma=0.14 \mathrm{~mm}$ and substitute the limiting value of $0.04 \mathrm{~mm}$ for $\sigma(\bar{X})$ :

$$
\begin{gathered}
\frac{0.14}{\sqrt{n}} \leq 0.04 \\
\sqrt{n} \geq \frac{0.14}{0.04}=3.5 \\
n \geq 12.25 \\
n=13
\end{gathered}
$$

As the sample size $n$ must be an integer, the minimum sample size is 13 .

\subsection{Central limit theorem}

\section{Theorem (The central limit theorem)}

If random and independent samples are taken from any practical population of mean $\mu$ and variance $\sigma^{2}$, as the sample size $n$ increases the distribution of sample means approaches a normal distribution:

$$
\bar{X} \sim N\left(\mu ; \sigma^{2} / n\right) \text { for } n \rightarrow \infty .
$$

\section{Remark}

- If the original population is normally distributed, means of samples of any size are normally distributed (and sums and differences of normally distributed variables are also normally distributed), if the original distribution is not normal, means of larger samples are closer to a normal distribution.

- Means of samples taken from almost all distributions encountered in practice will be normally distributed with negligible error if the sample size is at least 30 (almost the only exceptions will be samples taken from populations containing distant outliers). 
Example 40

A plant manufactures electric light bulbs with a burning life that is approximately normally distributed with a mean of 1200 hours and a standard deviation of 54 hours. Find the probability that a random sample of 36 bulbs will have a sample mean less than 1180 burning hours.

The bulb lives are normally distributed, so the mean of sample of size 36 is also normally distributed with the following characteristics:

$$
\begin{gathered}
\mu(\bar{X})=\mu=1200 \text { (hours) } \\
\sigma(\bar{X})=\frac{\sigma}{\sqrt{n}}=\frac{54}{\sqrt{36}}=9 \text { (hours). }
\end{gathered}
$$

Then the probability that a random sample of 16 bulbs will have a sample mean less than 1180 hours is:

$$
P(\bar{X}<1180)=\text { NORM.DIST }(1180 ; 1200 ; 9 ; 1) \doteq 0.0131
$$

\section{Exercise 41}

a) The mean content of a box of cat food is $2.50 \mathrm{~kg}$, and the standard deviation of the content of a box is $0.030 \mathrm{~kg}$. There are 24 boxes in a case, and there are 400 cases in a car load as it leaves the factory. What is the standard deviation of the amount of cat food contained in

i) a case,

ii) a car load?

b) A coffee dispensing machine is supposed to dispense a mean of 7.00 fluid ounces of coffee per cup with standard deviation of 0.25 fluid ounces. The distribution approximates a normal distribution. What is the probability that, when 12 cups are dispensed, their mean volume is more than 7.15 fluid ounces? 


\section{Statistical inferences for the mean}

\subsection{Hypothesis testing}

1. State the null hypothesis $H_{0}$ and the alternative hypothesis $H_{A}$ in terms of a population parameter.

2. State the test statistic TS and the level of significance of the test $p$ (it is usually 0.05 , sometimes 0.01 or 0.1 ). The quantity $1-p$ is called level of confidence.

3. Show calculations assuming that $H_{0}$ is true (compute the observed value of TS).

4. Compute the critical value and state the critical (rejection) region $C R$.

5. Find out whether the observed value of the test statistic belongs to the critical region $C R$ or not and state a conclusion.

\subsection{Inferences for the mean $-H_{0}: \mu=\mu_{0}$}

\section{Inferences for the mean when variance is known}

Test statistics

$$
\begin{gathered}
T S_{1}: z_{o b s}=\frac{x-\mu}{\sigma} \sim N(0 ; 1) \\
T S_{2}: z_{o b s}=\frac{\bar{x}-\mu}{\sigma} \sqrt{n} \sim N(0 ; 1)
\end{gathered}
$$

Critical value of the standard normal distribution $N(0 ; 1)$

- the value of $z_{\text {crit }}$ depends on the value of the stated level of significance $p$. We can find the value of $z_{\text {crit }}$ in statistical tables or compute it using Excel:

1. $z_{\text {crit }}=\operatorname{NORM.S.INV}(1-p / 2)$ for two-sided tests with $H_{A}: \mu \neq \mu_{0}$.

2. $z_{\text {crit }}=\operatorname{NORM.S.INV}(1-p)$ for one-sided tests with $H_{A}: \mu<\mu_{0}$ or $H_{A}: \mu>\mu_{0}$.

Critical (rejection) region

1. $C R=\left(-\infty,-z_{\text {crit }}\right) \cup\left(z_{\text {crit }},+\infty\right)$ for two-sided tests with $H_{A}: \mu \neq \mu_{0}$.

2. $C R=\left(-\infty,-z_{\text {crit }}\right)$ for one-sided tests with $H_{A}: \mu<\mu_{0}$.

3. $C R=\left(z_{\text {crit }},+\infty\right)$ for one-sided tests with $H_{A}: \mu>\mu_{0}$.

Conclusion:

$$
\begin{gathered}
z_{\text {obs }} \in C R \Rightarrow H_{0} \text { is rejected } \\
z_{\text {obs }} \notin C R \Rightarrow H_{0} \text { is not rejected }
\end{gathered}
$$

\section{Example 42}

It is important that a certain solution has a $\mathrm{pH}$ of 8.30 . The method used gives measurements which are approximately normally distributed about the actual $\mathrm{pH}$ of the solution with a known standard deviation of 0.020 . Is there evidence at the $5 \%$ level of significance that the mean $\mathrm{pH}$ has changed, in case a single determination shows $\mathrm{pH}$ of 8.32 ? 
The population standard deviation is known and we have only single determination $x$, so we use the formula for $T S_{1}$. We are concerned with possible changes of population mean in both directions, so we use a two-sided test of significance:

1. Stating the null and the alternative hypothesis:

$$
\begin{gathered}
H_{0}: \mu=8.30 \\
H_{A}: \mu \neq 8.30
\end{gathered}
$$

2. Stating the test statistic TS:

$$
T S_{1}: z=\frac{x-\mu}{\sigma}
$$

and the level of significance of the test $p$ :

$$
p=0.05
$$

3. Showing calculations assuming that $H_{0}$ is true, i.e. computing the observed value of the test statistic $z_{o b s}$ :

$$
z_{\text {obs }}=\frac{8.32-8.30}{0.020}=1
$$

4. Computing the critical value of the corresponding distribution $z_{\text {crit }}$ :

$$
z_{\text {crit }}=\operatorname{NORM.S.INV}(1-p / 2)=\operatorname{NORM} . S . I N V(0.975) \doteq 1.96
$$

and stating the critical (rejection) region $C R$ :

$$
C R=\left(-\infty,-z_{\text {crit }}\right) \cup\left(z_{\text {crit }},+\infty\right)=(-\infty,-1.96) \cup(1.96,+\infty)
$$

5. Finding out whether the observed value of the test statistic $z_{o b s}$ belongs to the critical region $C R$ and stating conclusion:

$$
z_{\text {obs }} \notin C R \Rightarrow H_{0} \text { is not rejected }
$$

We do not have enough evidence from this calculation to say that the $\mathrm{pH}$ is not equal to 8.30. We could say that the difference from a $\mathrm{pH}$ of 8.30 is not statistically significant at the $5 \%$ level of significance.

Example 43

It is very important that a certain solution has a $\mathrm{pH}$ of 8.30 . The method used gives measurements which are approximately normally distributed about the actual $\mathrm{pH}$ of the solution with a known standard deviation of 0.020 . Is there evidence at the $5 \%$ level of significance that the mean $\mathrm{pH}$ has changed, in case a sample of 4 determinations shows $\mathrm{pH}$ of $8.31,8.34,8.32,8.31$ ?

The population standard deviation is known and we have a sample of 4 determinations $x_{1}, \ldots, x_{4}$, so we use the formula for $T S_{2}$. We are concerned with possible changes of population mean in both directions, so we use a two-sided test of significance: 
1. Stating the null and the alternative hypothesis:

$$
\begin{aligned}
& H_{0}: \mu=8.30 \\
& H_{A}: \mu \neq 8.30
\end{aligned}
$$

2. Stating the test statistic TS:

$$
T S_{2}: z=\frac{\bar{x}-\mu}{\sigma} \sqrt{n}
$$

and the level of significance of the test $p$ :

$$
p=0.05
$$

3. Showing calculations assuming that $H_{0}$ is true, i.e. computing the observed value of the test statistic $z_{o b s}$ :

$$
\begin{gathered}
\bar{x}=\frac{1}{N} \sum_{i=1}^{N} x_{i}=\frac{1}{4}(8.31+8.34+8.32+8.31)=8.32 \\
z_{o b s}=\frac{8.32-8.30}{0.020} \sqrt{4}=2
\end{gathered}
$$

4. Computing the critical value of the corresponding distribution $z_{\text {crit }}$ :

$$
z_{\text {crit }}=\operatorname{NORM} . S . I N V(1-p / 2)=\operatorname{NORM} . S . I N V(0.975) \doteq 1.96
$$

and stating the critical (rejection) region $C R$ :

$$
C R=\left(-\infty,-z_{\text {crit }}\right) \cup\left(z_{\text {crit }},+\infty\right)=(-\infty,-1.96) \cup(1.96,+\infty)
$$

5. Finding out whether the observed value of the test statistic $z_{o b s}$ belongs to the critical region $C R$ and stating conclusion:

$$
z_{\text {obs }} \in C R \Rightarrow H_{0} \text { is rejected }
$$

Since $z_{o b s} \in C R$, we reject the null hypothesis and accept the alternative hypothesis $\mu \neq$ 8.30. At the $5 \%$ level of significance we conclude that the true mean $\mathrm{pH}$ is no longer 8.30 (the evidence against the null hypothesis is stronger now, because we have a sample of four determinations with the mean of 8.32 instead of only one determination with the value of 8.32).

\section{Inferences for the mean when variance is estimated from a sample}

Test statistics

$$
T S: t=\frac{\bar{x}-\mu}{s} \sqrt{n} \sim t(n-1)
$$

Critical value is a quantile of $t$-distribution with $n-1$ degrees of freedom Excel:

1. $t_{c r i t}=\mathrm{T} . \operatorname{INV}(1-p / 2 ; n-1)$ for two-sided tests with $H_{A}: \mu \neq \mu_{0}$.

2. $t_{c r i t}=\mathrm{T} . \mathrm{INV}(1-p ; n-1)$ for one-sided tests with $H_{A}: \mu<\mu_{0}$ or $H_{A}: \mu>\mu_{0}$. 
Critical (rejection) region

1. $C R=\left(-\infty,-t_{\text {crit }}\right) \cup\left(t_{\text {crit }},+\infty\right)$ for two-sided tests with $H_{A}: \mu \neq \mu_{0}$.

2. $C R=\left(-\infty,-t_{\text {crit }}\right)$ for one-sided tests with $H_{A}: \mu<\mu_{0}$.

3. $C R=\left(t_{\text {crit }},+\infty\right)$ for one-sided tests with $H_{A}: \mu>\mu_{0}$.

Conclusion:

$$
\begin{gathered}
z_{\text {obs }} \in C R \Rightarrow H_{0} \text { is rejected } \\
z_{\text {obs }} \notin C R \Rightarrow H_{0} \text { is not rejected }
\end{gathered}
$$

\section{Example 44}

The electrical resistances of components are measured as they are produced. A sample of six items gives a sample mean of $2.62 \mathrm{ohms}$ and a sample standard deviation of $0.121 \mathrm{ohms}$. Is there evidence at the $1 \%$ level of significance that the population mean is significantly less than 2.80 ohms?

Tests of significance:

1. Stating the null and the alternative hypothesis:

$$
\begin{aligned}
& H_{0}: \mu=2.80 \\
& H_{A}: \mu<2.80
\end{aligned}
$$

2. Stating the test statistic TS:

$$
T S: t=\frac{\bar{x}-\mu}{s} \sqrt{n} \sim t(n-1)
$$

and the level of significance of the test $p$ :

$$
p=0.01
$$

3. Showing calculations assuming that $H_{0}$ is true, i.e. computing the observed value of the test statistic $t_{o b s}$ :

$$
t_{\text {obs }}=\frac{2.62-2.80}{0.121} \sqrt{6} \doteq-3.64
$$

4. Computing the critical value of the corresponding distribution $t_{\text {crit }}$ :

$$
t_{\text {crit }}=\mathrm{T} . \operatorname{INV}(1-p ; n-1)=\mathrm{T} . \operatorname{INV}(0.99 ; 5) \doteq 3.36
$$

and stating the critical (rejection) region $C R$ :

$$
C R=\left(-\infty,-t_{\text {crit }}\right)=(-\infty,-3.36)
$$

5. Finding out whether the observed value of the test statistic $t_{o b s}$ belongs to the critical region $C R$ and stating conclusion:

$$
t_{\text {obs }} \in C R \Rightarrow H_{0} \text { is rejected }
$$

Since $t_{o b s} \in C R$, we reject the null hypothesis and accept the alternative hypothesis $\mu<$ 2.80. At the $1 \%$ level of significance we conclude that the true mean electrical resistance of components is less than 2.80 ohms. 


\subsection{Confidence interval for the mean}

A population mean is an uncertain value for which we need an estimate. The sample mean gives a point estimate for the population mean but we often need an interval estimate. That interval corresponds to a stated level of confidence that the interval contains the true population mean.

Confidence interval for the mean when the population variance $\sigma$ is known:

$$
\mu \in\left(\bar{x}-z_{c r i t} \frac{\sigma}{\sqrt{n}} ; \bar{x}+z_{c r i t} \frac{\sigma}{\sqrt{n}}\right)
$$

Remark

- $z_{\text {crit }}$ is a critical value of the standardized normal distribution $N(0 ; 1)$, which depends on the value of the stated level of confidence $1-p$

- it can be computed by Excel: $z_{\text {crit }}=\operatorname{NORM} \cdot \operatorname{S.INV}(1-p / 2)$

Confidence interval for the mean when the population variance $\sigma$ is not known:

$$
\mu \in\left(\bar{x}-t_{c r i t} \frac{s}{\sqrt{n}} ; \bar{x}+t_{c r i t} \frac{s}{\sqrt{n}}\right)
$$

Remark

- $t_{\text {crit }}$ is a critical value of $t$-distribution with $n-1$ degrees of freedom $t(n-1)$, which depends on the value of the stated level of confidence $1-p$

- it can be computed by Excel: $t_{c r i t}=\operatorname{T} \cdot \operatorname{INV}(1-p / 2 ; n-1)$

\section{Example 45}

A certain dimension is measured on four successive items coming off a production line. This sample gives a sample mean $\bar{x}=2.384$ and a sample standard deviation $s=0.048$.

a) On the basis of this sample, state the $95 \%$ confidence interval for the population mean.

b) State the $95 \%$ confidence interval for the population mean in case we knew the true standard deviation was 0.048 .

a) we use the formula for confidence interval for the mean when the population variance $\sigma$ is not known:

$$
\mu \in\left(\bar{x}-t_{\text {crit }} \frac{s}{\sqrt{n}} ; \bar{x}+t_{\text {crit }} \frac{s}{\sqrt{n}}\right)
$$

where $n=4, \bar{x}=2.384, s=0.048, p=0.05, t_{\text {crit }}=\operatorname{T} . \operatorname{INV}(0.975 ; 3) \doteq 3.182$

$$
\mu \in(2.31 ; 2.46)
$$


b) we use the formula for confidence interval for the mean when the population variance $\sigma$ is known:

$$
\mu \in\left(\bar{x}-z_{\text {crit }} \frac{\sigma}{\sqrt{n}} ; \bar{x}+z_{\text {crit }} \frac{\sigma}{\sqrt{n}}\right)
$$

where $n=4, \bar{x}=2.384, \sigma=0.048, p=0.05, z_{\text {crit }}=\operatorname{NORM} . \operatorname{S.INV}(0.975) \doteq 1.960$

$$
\mu \in(2.34 ; 2.43)
$$

\subsection{Comparison of sample means - $H_{0}: \mu_{1}=\mu_{2}$}

\section{Independent samples $t$-test}

Test statistic

$$
T S: t=\frac{\bar{x}_{1}-\bar{x}_{2}}{\sqrt{\frac{s_{1}^{2}\left(n_{1}-1\right)+s_{2}^{2}\left(n_{2}-1\right)}{\left(n_{1}-1\right)+\left(n_{2}-1\right)}\left(\frac{1}{n_{1}}+\frac{1}{n_{2}}\right)}} \sim t\left(n_{1}-1+n_{2}-1\right)
$$

Critical value is a quantile of $t$-distribution with $n_{1}+n_{2}-2$ degrees of freedom Excel:

1. $t_{c r i t}=\mathrm{T} . \mathrm{INV}\left(1-p / 2 ; n_{1}+n_{2}-2\right)$ for two-sided tests with $H_{A}: \mu_{1} \neq \mu_{2}$.

2. $t_{c r i t}=\operatorname{T.INV}\left(1-p ; n_{1}+n_{2}-2\right)$ for one-sided tests with $H_{A}: \mu_{1}<\mu_{2}$ or $H_{A}: \mu_{1}>\mu_{2}$.

Critical (rejection) region

1. $C R=\left(-\infty,-t_{\text {crit }}\right) \cup\left(t_{\text {crit }},+\infty\right)$ for two-sided tests with $H_{A}: \mu_{1} \neq \mu_{2}$.

2. $C R=\left(-\infty,-t_{\text {crit }}\right)$ for one-sided tests with $H_{A}: \mu_{1}<\mu_{2}$.

3. $C R=\left(t_{\text {crit }},+\infty\right)$ for one-sided tests with $H_{A}: \mu_{1}>\mu_{2}$.

Conclusion:

$$
\begin{gathered}
z_{\text {obs }} \in C R \Rightarrow H_{0} \text { is rejected } \\
z_{\text {obs }} \notin C R \Rightarrow H_{0} \text { is not rejected }
\end{gathered}
$$

\section{Example 46}

Two methods of determining the nickel content of steel are compared using four determinations by each method. The results are:

For method 1: $\bar{x}_{1}=3.285, s_{1}=0.00774$

For method 2: $\bar{x}_{2}=3.258, s_{2}=0.00960$

Assuming that the two estimates of variance are compatible, is the difference in means statistically significant at the $5 \%$ level of significance?

Tests of significance:

1. Stating the null and the alternative hypothesis:

$$
\begin{aligned}
& H_{0}: \mu_{1}=\mu_{2} \\
& H_{A}: \mu_{1} \neq \mu_{2}
\end{aligned}
$$


2. Stating the test statistic TS:

$$
T S: t=\frac{\bar{x}_{1}-\bar{x}_{2}}{\sqrt{\frac{s_{1}^{2}\left(n_{1}-1\right)+s_{2}^{2}\left(n_{2}-1\right)}{\left(n_{1}-1\right)+\left(n_{2}-1\right)}\left(\frac{1}{n_{1}}+\frac{1}{n_{2}}\right)}}
$$

and the level of significance of the test $p$ :

$$
p=0.05
$$

3. Showing calculations assuming that $H_{0}$ is true, i.e. computing the observed value of the test statistic $t_{o b s}$ :

$$
t_{o b s}=\frac{3.285-3.258}{\sqrt{\frac{0.00774^{2}(4-1)+0.00960^{2}(4-1)}{(4-1)+(4-1)}\left(\frac{1}{4}+\frac{1}{4}\right)}} \doteq 4.38
$$

4. Computing the critical value of the corresponding distribution $t_{c r i t}$ :

$$
t_{\text {crit }}=T . \operatorname{INV}\left(1-p / 2 ; n_{1}+n_{2}-2\right)=T . \operatorname{INV}(0.975 ; 6) \doteq 2.45
$$

and stating the critical (rejection) region $C R$ :

$$
C R=\left(-\infty,-t_{\text {crit }}\right) \cup\left(t_{\text {crit }},+\infty\right)=(-\infty,-2.45) \cup(2.45,+\infty)
$$

5. Finding out whether the observed value of the test statistic $t_{\text {obs }}$ belongs to the critical region $C R$ and stating conclusion:

$$
t_{\text {obs }} \in C R \Rightarrow H_{0} \text { is rejected }
$$

Since $t_{o b s} \in C R$, we reject the null hypothesis and accept the alternative hypothesis $\mu_{1} \neq$ $\mu_{2}$. The difference in means is statistically significant at the $5 \%$ level of significance.

\section{Paired samples $t$-test}

Requirements:

- the two random samples are dependent and have the same number of observations $\left(n_{1}=\right.$ $\left.n_{2}=n\right)$

- each observation from the first sample $\left(x_{i}\right)$ forms a pair with one observation from the second sample $\left(y_{i}\right)$

Tests of significance

- for all pairs we compute pair differences $d\left(d_{i}=x_{i}-y_{i}\right)$

Test statistic

$$
T S: t=\frac{\bar{d}-0}{s_{d}} \sqrt{n} \sim t(n-1)
$$

Critical value is a quantile of $t$-distribution with $n-1$ degrees of freedom Excel:

1. $t_{c r i t}=\mathrm{T} . \mathrm{INV}(1-p / 2 ; n-1)$ for two-sided tests with $H_{A}: \mu_{1} \neq \mu_{2}$.

2. $t_{\text {crit }}=\mathrm{T} . \mathrm{INV}(1-p ; n-1)$ for one-sided tests with $H_{A}: \mu_{1}<\mu_{2}$ or $H_{A}: \mu_{1}>\mu_{2}$.

Critical (rejection) region 
1. $C R=\left(-\infty,-t_{\text {crit }}\right) \cup\left(t_{\text {crit }},+\infty\right)$ for two-sided tests with $H_{A}: \mu_{1} \neq \mu_{2}$.

2. $C R=\left(-\infty,-t_{\text {crit }}\right)$ for one-sided tests with $H_{A}: \mu_{1}<\mu_{2}$.

3. $C R=\left(t_{\text {crit }},+\infty\right)$ for one-sided tests with $H_{A}: \mu_{1}>\mu_{2}$.

Conclusion:

$$
\begin{gathered}
z_{\text {obs }} \in C R \Rightarrow H_{0} \text { is rejected } \\
z_{\text {obs }} \notin C R \Rightarrow H_{0} \text { is not rejected }
\end{gathered}
$$

\section{Example 47}

We decide to run a test using an experimental evaporation pan and a standard evaporation pan over ten successive days. The two types are set up side-by-side so that atmospheric conditions should be the same. A coin is tossed to decide which evaporation pan is on the lefthand side and which on the righthand side on any particular day. The measured daily evaporations are as follows.

Evaporation (mm):

Pan A: 9.1, 4.6, 14.0, 16.9, 11.4, 10.7, 27.4, 22.8, 42.8, 29.4

Pan B: 6.7, 3.1, 13.8, 16.6, 12.3, 6.5, 24.2, 20.1, 41.9, 27.7

Does the experimental Pan A give significantly higher evaporation than the standard Pan $\mathrm{B}$ at the $1 \%$ level of significance?

First we have to compute the differences $d_{i}$ (Pan A - Pan B):

$d_{i}: 2.4,1.5,0.2,0.3,-0.9,4.2,3.2,2.7,0.9,1.7$

Tests of significance:

1. Stating the null and the alternative hypothesis:

$$
\begin{aligned}
& H_{0}: \mu_{d}=0 \\
& H_{A}: \mu_{d}>0
\end{aligned}
$$

2. Stating the test statistic TS:

$$
T S: t=\frac{\bar{d}-0}{s_{d}} \sqrt{n}
$$

and the level of significance of the test $p$ :

$$
p=0.01
$$

3. Showing calculations assuming that $H_{0}$ is true, i.e. computing the observed value of the test statistic $t_{o b s}$ :

$$
\begin{gathered}
\bar{d}=\frac{1}{n} \sum_{i=1}^{n} d_{i}=\frac{1}{10}(2.4+1.5+0.2+0.3-0.9+4.2+3.2+2.7+0.9+1.7)=1.62 \\
s_{d}=\sqrt{\frac{1}{N-1} \sum_{i=1}^{N}\left(d_{i}-\bar{d}\right)^{2}} \doteq 1.55 \\
t_{o b s}=\frac{\bar{d}-0}{s_{d}} \sqrt{n} \doteq 3.31
\end{gathered}
$$


4. Computing the critical value of the corresponding distribution $t_{\text {crit }}$ :

$$
t_{\text {crit }}=\mathrm{T} . \mathrm{INV}(1-p ; n-1)=\mathrm{T} . \mathrm{INV}(0.99 ; 9) \doteq 2.82
$$

and stating the critical (rejection) region $C R$ :

$$
C R=\left(t_{\text {crit }},+\infty\right)=(2.82,+\infty)
$$

5. Finding out whether the observed value of the test statistic $t_{o b s}$ belongs to the critical region $C R$ and stating conclusion:

$$
t_{\text {obs }} \in C R \Rightarrow H_{0} \text { is rejected }
$$

Since $t_{o b s} \in C R$, we reject the null hypothesis and accept the alternative hypothesis $\mu_{d}>0$. The experimental Pan A give significantly higher evaporation than the standard Pan B at the $1 \%$ level of significance.

\section{Exercise 48}

a) A cocoa packaging machine fills bags so that the bag contents have a standard deviation of $3.5 \mathrm{~g}$. Weights of contents of bags are normally distributed. If a random sample of 20 bags gives a mean of $102.0 \mathrm{~g}$, what is the $99 \%$ confidence interval for the mean weight of the population (i.e., all bags)?

b) Benzene in the air workers breathe can cause cancer. It is very important for the benzene content of air in a particular plant to be not more than $1.00 \mathrm{ppm}$. Samples are taken to check the benzene content of the air. 25 specimens of air from one location in the plant gave a mean content of $0.760 \mathrm{ppm}$, and the standard deviation of benzene content was estimated on the basis of the sample to be $0.45 \mathrm{ppm}$. Benzene contents in this case are found to be normally distributed.

i) Is there evidence at the $1 \%$ level of significance that the true mean benzene content is less than or equal to $1.00 \mathrm{ppm}$ ?

ii) Find the $95 \%$ confidence interval for the true mean benzene content.

c) Two chemical processes for manufacturing the same product are being compared under the same conditions. Yield from Process A gives an average value of 96.2 from six runs, and the estimated standard deviation of yield is 2.75. Yield from Process B gives an average value of 93.3 from seven runs, and the estimated standard deviation is 3.35. Yields follow a normal distribution. Is the difference between the mean yields statistically significant? Use the $5 \%$ level of significance. 


\section{Regression and correlation analysis}

The goal of regression analysis is to describe the relationship between two variables based on observed data and to predict the value of the dependent variable $y$ based on the value of the independent variable $x$. To better visualize the association between two data sets $x_{1}, \ldots, x_{n}$ and $y_{1}, \ldots, y_{n}$ we can employ a chart called a scatter diagram (also called a scatter plot).

The relationship can be represented by a simple equation called the regression equation.

\section{Definition}

Let $\left(x_{i}, y_{i}\right), i=1, \ldots, n$ be $n$ pairs of observations. The simple regression linear model of $y$ on $x$ is

$$
y=\beta_{0}+\beta_{1} x_{i}+\epsilon,
$$

where $\beta_{0}$ and $\beta_{1}$ are constant parameters (called regression coefficients) that we want to estimate and $\epsilon$ is the random error term.

\subsection{Method of least squares}

In practice we will build the linear regression model from the sample data using the least squares method. Thus we seek coefficients $a$ and $b$ such that

$$
\hat{y}_{i}=a+b x_{i}
$$

where $a, b$ are some estimates for the model coefficients $\beta_{0}, \beta_{1}$ and $\hat{y}_{i}$ is the estimated (predicted) $y$ value for $i$-th observation $x_{i}$.

The difference between the observed value $y_{i}$ and the predicted value $\hat{y}_{i}$ is called as a residual. The $i$-th residual is defined as

$$
e_{i}=y_{i}-\hat{y}_{i}=y_{i}-\left(a+b x_{i}\right) .
$$

The best fit line (regression line) is the line for which the sum of the distances between each of the $n$ data points and the line is as small as possible. A mathematically useful approach is therefore to find the line with the property that the sum of the following squares is minimal.

So, $a, b$ are obtained by finding the values that minimize the sum of the squared differences between $y$ and $\hat{y}$. This sum of the squares of the errors (residuals) for all $n$ points is abbreviated as SSE.

$$
\min \sum_{i} e_{i}^{2}=\min \sum_{i}\left(y_{i}-\hat{y}_{i}\right)^{2}=\min \sum_{i}\left(y_{i}-\left(a+b x_{i}\right)\right)^{2}
$$

To minimize a quantity we take the derivative with respect to the independent variable and set it equal to zero.

In this case there are two independent variables, $a$ and $b$, so we take partial derivatives with respect to each of them and set the derivatives equal to zero. We have

$$
\frac{\partial(S S E)}{\partial a}=\frac{\partial}{\partial a} \sum_{i}\left(y_{i}-\left(a+b x_{i}\right)\right)^{2}=-2\left(\sum_{i} y_{i}-n a-b \sum_{i} x_{i}\right)=0
$$


and

$$
\frac{\partial(S S E)}{\partial b}=\frac{\partial}{\partial b} \sum_{i}\left(y_{i}-\left(a+b x_{i}\right)\right)^{2}=-2\left(\sum_{i} x_{i} y_{i}-a \sum_{i} x_{i}-b \sum_{i} x_{i}^{2}\right)=0 .
$$

We get the least squares normal equations

$$
n a+b \sum_{i} x_{i}=\sum_{i} y_{i}
$$

and

$$
a \sum_{i} x_{i}+b \sum_{i} x_{i}^{2}=\sum_{i} x_{i} y_{i}
$$

The solution to the normal equations results in the least squares estimators

$$
a=\frac{\sum_{i} y_{i}-b \sum_{i} x_{i}}{n}=\bar{y}-b \bar{x}
$$

and

$$
b=\frac{\sum_{i} x_{i} y_{i}-\frac{1}{n}\left(\sum_{i} x_{i} \sum_{i} y_{i}\right)}{\sum_{i} x_{i}^{2}-\frac{1}{n}\left(\sum_{i} x_{i}\right)^{2}}=\frac{\sum_{i}\left(x_{i}-\bar{x}\right)\left(y_{i}-\bar{y}\right)}{\sum_{i}\left(x_{i}-\bar{x}\right)^{2}}
$$

Remark

Excel:

Insert $\rightarrow$ Charts $\mid$ Scatter $\rightarrow$ Layout

and then "Linear Trend"

Example 49

The table shows the results of two different tests for 8 students

\begin{tabular}{l|llllllll}
\hline 1st test & 80 & 50 & 36 & 58 & 72 & 60 & 56 & 68 \\
\hline 2nd test & 65 & 60 & 35 & 39 & 48 & 44 & 48 & 61 \\
\hline
\end{tabular}

Find the best-fit regression equation of $y$ (2nd test) on $x$ (1st test).

We have 8 points $(n=8)$. We calculate the following sums:

$$
\sum_{i=1}^{8} x_{i}=480, \sum_{i=1}^{8} y_{i}=400, \sum_{i=1}^{8} x_{i}^{2}=30104, \sum_{i=1}^{8} x_{i} y_{i}=24654
$$

The centroidal point is given by $\bar{x}=60, \bar{y}=50$.

Then

$$
b=\frac{\sum_{i} x_{i} y_{i}-\frac{1}{n}\left(\sum_{i} x_{i} \sum_{i} y_{i}\right)}{\sum_{i} x_{i}^{2}-\frac{1}{n}\left(\sum_{i} x_{i}\right)^{2}}=\frac{24654-\frac{1}{8}(480 \cdot 400)}{30104-\frac{1}{8}(480)^{2}}=0.5015
$$

and

$$
a=\bar{y}-b \bar{x}=50-0.5015 \cdot 60=19.908
$$

The regression equation is

$$
y=19.908+0.5015 x
$$




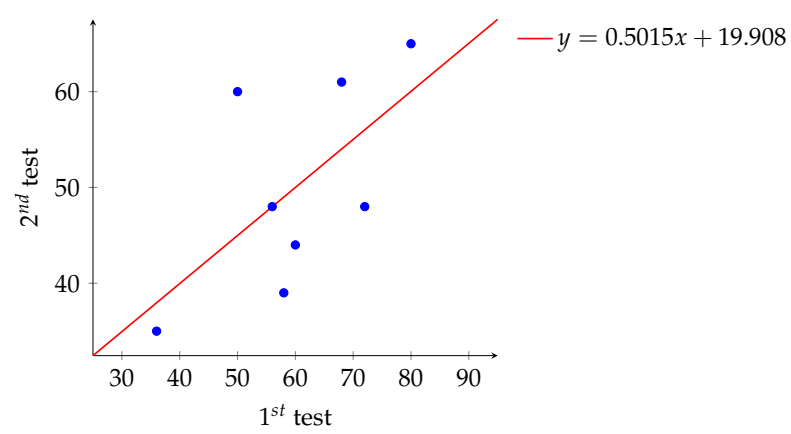

Exercise 50

The shear resistance of soil $(y)$ is determined by measurements as a function of the normal stress $(x)$. The data are shown in the table:

\begin{tabular}{c|cccccccc}
\hline$x\left(\mathrm{kN} \mathrm{m}^{-2}\right)$ & 10 & 12 & 13 & 16 & 17 & 18 & 20 & 21 \\
\hline$y\left(\mathrm{kN} \mathrm{m}^{-2}\right)$ & 14.08 & 16.94 & 17.68 & 20.68 & 21.72 & 22.80 & 24.79 & 25.67 \\
\hline
\end{tabular}

Find the regression line of $y$ on $x$. Plot the data, the regression line and the centroidal point.

\subsection{Regression model validation}

Coefficient of Determination ( $R$ Squared) $R^{2}$ is used to analyze how differences in one variable can be explained by a difference in a second variable. The range is 0 to 1 (i.e. $0 \%$ to $100 \%$ of the variation in $y$ can be explained by the $x$-variable).

The higher value R-squared means the better model. Clearly $0 \leq R^{2} \leq 1$, so a value of $R^{2}$ closer to one indicates the better fit and value of $R^{2}$ closer to zero indicates the poor fit.

We compute the R-squared as a fraction of the variances:

$$
R^{2}=\frac{S_{R}^{2}}{S_{T}^{2}}=1-\frac{S S E}{S_{T}^{2}}
$$

where

$$
\begin{aligned}
S_{T}^{2} & =\sum_{i=1}^{n}\left(y_{i}-\bar{y}\right)^{2} \quad \text { total sum of squares, } \\
S_{R}^{2} & =\sum_{i=1}^{n}\left(\tilde{y}_{i}-\bar{y}\right)^{2} \quad \text { regression sum of squares, } \\
S S E & =\sum_{i=1}^{n}\left(y_{i}-\tilde{y}_{i}\right)^{2} \quad \text { residual sum of squares. }
\end{aligned}
$$

Total variation is made up of two parts:

$$
S_{T}^{2}=S_{R}^{2}+S S E
$$

Adjusted Coefficient of Determination $R_{a d j}^{2}$ is an adjustment for the R-squared that takes into account the number of variables in a data set. 
The formula is:

$$
R_{a d j}^{2}=1-\left(1-R^{2}\right) \frac{n-1}{n-p-1},
$$

where $n$ is the number of points in the data sample and $p$ is the number of independent regressors, i.e. the number of variables in the model, excluding the constant.

Residual Standard Deviation $R_{r e s}$ is the standard deviation of the residual values, or the difference between a set of observed and predicted values. The standard deviation of the residuals calculates how much the data points spread around the regression line. The result is used to measure the error of the regression line's predictability.

The formula is:

$$
R_{\text {res }}=\sqrt{\frac{1}{n-2} S S E}=\sqrt{\frac{1}{n-2} \sum_{i=1}^{n}\left(y_{i}-\tilde{y}_{i}\right)^{2}},
$$

where $n$ is the number of points in the data sample.

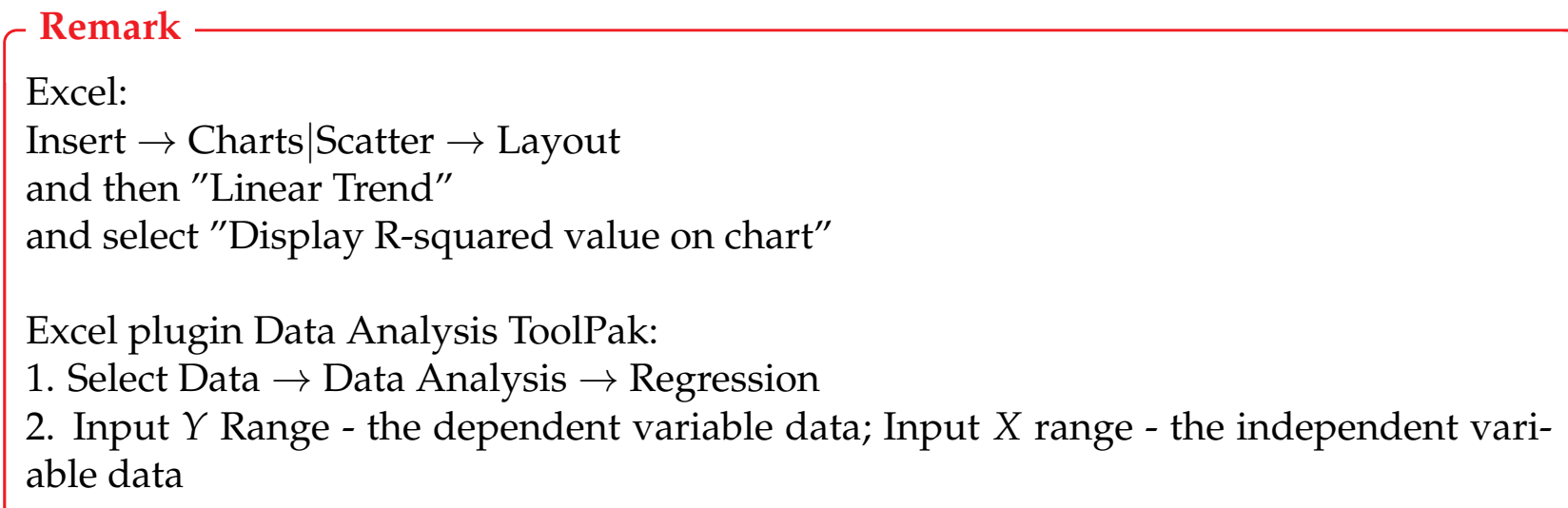

\section{Example 51}

We examine the relationship between the selling price of a house and its size.

\begin{tabular}{l|ccccccc}
\hline price (CZK 1000s) & 4287 & 5460 & 4882 & 5390 & 3482 & 3820 & 5670 \\
\hline size $\mathrm{m}^{2}$ & 130 & 148 & 160 & 175 & 100 & 140 & 200 \\
\hline
\end{tabular}

Find the linear regression equation of the price on size of the house. Calculate a coefficient of determination for the data.

We can chart a regression in Excel.

- Insert $\rightarrow$ Charts $\mid$ Scatter

- To add a regression line, choose "Layout" from the "Chart Tools" menu

- In the dialog box, select "Trendline" and then "Linear Trendline"

- To add the $R^{2}$ value, select "Display R-squared value on chart" 


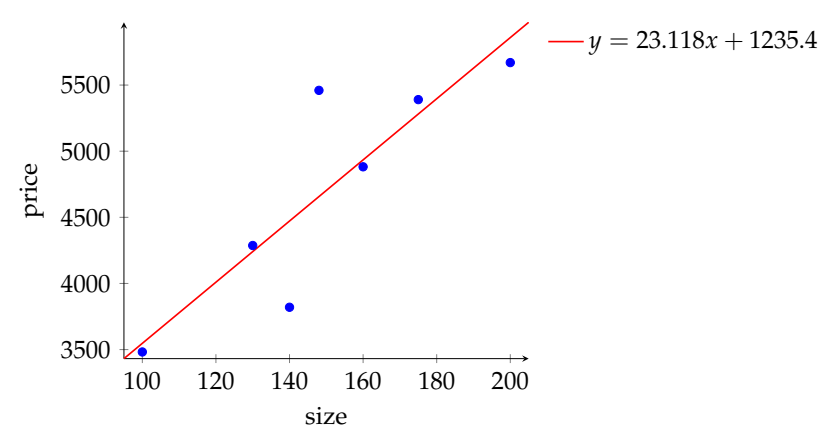

The regression equation is

$$
y=1235.4+23.118 x
$$

and the coefficient of determination is

$$
R^{2}=0.7471,
$$

so, $74.71 \%$ of the variation in house prices is explained by variation in its size.

\section{Exercise 52}

Consider the data obtained from a chemical process where the yield of the process is thought to be related to the reaction temperature.

\begin{tabular}{l|cccccccc}
\hline temperature & 50 & 54 & 56 & 62 & 67 & 72 & 75 & 79 \\
\hline yield & 122 & 128 & 125 & 144 & 149 & 167 & 162 & 175 \\
\hline
\end{tabular}

Find the regression line of the yield of the process on the reaction temperature. Plot the data, the regression line and calculate a coefficient of determination.

\subsection{Inferences for coefficients}

The standard error of an estimator reflects how it varies under repeated sampling. We have:

$$
\begin{aligned}
s_{b} & =\frac{R_{r e s}}{\sqrt{\sum_{i=1}^{n}\left(x_{i}-\bar{x}\right)^{2}}} \\
s_{a}^{2} & =R_{r e s}^{2}\left[\frac{1}{n}+\frac{\bar{x}^{2}}{\sum_{i=1}^{n}\left(x_{i}-\bar{x}\right)^{2}}\right]
\end{aligned}
$$

The standard errors can be used to compute confidence intervals, these standard errors are then multiplied by appropriate values of $t$-distribution with $n-2$ degrees of freedom, $t_{\alpha}$, to find confidence intervals.

There is approximately $(1-\alpha) \%$ chance that the intervals

$$
\begin{aligned}
& {\left[a-t_{\alpha} s_{a}, a+t_{\alpha} s_{a}\right]} \\
& {\left[b-t_{\alpha} s_{b}, b+t_{\alpha} s_{b}\right]}
\end{aligned}
$$

contain the true values of $\beta_{0}, \beta_{1}$. 
The standard errors can also be used to perform hypothesis test on the coefficients.

\section{$t$-test for a population slope}

$$
\begin{aligned}
& H_{0}: \beta_{1}=0 \text { there is no linear relationship between } x \text { and } y \\
& H_{1}: \beta_{1} \neq 0 \text { there is some linear relationship between } x \text { and } y
\end{aligned}
$$

To test the null hypothesis, we compute a $t$-statistic, given by

$$
t=\frac{b-\beta_{1}}{s_{b}}
$$

This will have a $t$-distribution with $(n-2)$ degrees of freedom.

\section{Remark}

Excel plugin Data Analysis ToolPak:

1. Select Data $\rightarrow$ Data Analysis $\rightarrow$ Regression

2. Input $Y$ Range - the dependent variable data; Input $X$ range - the independent variable data

and select "Confidence level: $1-\alpha \%$ "

\section{Example 53}

We examine the relationship between the selling price of a house and its size. Compute the $95 \%$ confidence interval for regression coefficient $b$. Is there a linear relationship between the selling price of a house and its size? Use $t$-test for a population slope.

\begin{tabular}{|c|c|c|c|c|c|c|}
\hline \multicolumn{2}{|c|}{ Regression Statistics } & & & & & \\
\hline Multiple R & 0,864341111 & & & & & \\
\hline R Square & 0,747085556 & & & & & \\
\hline Adjusted R Square & 0,696502667 & & & & & \\
\hline Standard Error & 474,7088473 & & & & & \\
\hline Observations & 7 & & & & & \\
\hline \multicolumn{7}{|l|}{ ANOVA } \\
\hline & $d f$ & SS & MS & $F$ & Significance $F$ & \\
\hline Regression & 1 & 3328291,551 & 3328292 & 14,76953 & 0,012085361 & \\
\hline Residual & 5 & 1126742,449 & 225348,5 & & & \\
\hline \multirow[t]{2}{*}{ Total } & 6 & 4455034 & & & & \\
\hline & Coefficients & Standard Error & t Stat & P-value & Lower 95\% & Upper $95 \%$ \\
\hline Intercept & 1235,423659 & 922,501617 & 1,33921 & 0,238154 & $-1135,942241$ & 3606,789559 \\
\hline X Variable 1 & 23,11779144 & 6,015378871 & 3,843115 & 0,012085 & 7,65476778 & 38,5808151 \\
\hline
\end{tabular}

\begin{tabular}{l|ccccccc}
\hline price (CZK 1000s) & 4287 & 5460 & 4882 & 5390 & 3482 & 3820 & 5670 \\
\hline size $\mathrm{m}^{2}$ & 130 & 148 & 160 & 175 & 100 & 140 & 200 \\
\hline
\end{tabular}

SUMMARY OUTPUT 
We see, that

$$
\begin{aligned}
b & =23.118 \\
s_{b} & =6.01 \\
t & =3.84 \\
p & =0.01
\end{aligned}
$$

$t_{\alpha}$ is computed by the formula $=T \cdot \operatorname{INV}(1-\alpha ; n-2)$,

the $95 \%$ confidence interval for $b$ is $[23.118-2.5716 .01,23.118+2.5716 .01]=[7.65,38.56]$

Now, we find, if there is a linear relationship.

$$
\begin{aligned}
& H_{0}: \beta_{1}=0 \\
& H_{1}: \beta_{1} \neq 0
\end{aligned}
$$

We have two options for decision.

- $t=3.84>2.57=t_{\alpha} \Rightarrow H_{0}$ is rejected

- $p=0.01 \Rightarrow p$-value is less than the chosen significance level then we can reject the null hypothesis

There is sufficient evidence that the size of house affects house price.

\subsection{Correlation}

Correlation is a measure of the association between two random variables, say $X$ and $Y$. We do assume for this analysis that $X$ and $Y$ are related linearly, so the usual correlation coefficient gives a measure of the linear association between $X$ and $Y$. In practice we work with the sample correlation coefficient $r$. It is also called the Pearson correlation coefficient. The symbol for the population correlation coefficient is $\rho$.

This is calculated as

$$
r=\frac{\sum_{i}\left[\left(x_{i}-\bar{x}\right)\left(y_{i}-\bar{y}\right)\right]}{\sum_{i}\left(x_{i}-\bar{x}\right) \cdot \sum_{i}\left(y_{i}-\bar{y}\right)},
$$

which can be shown to be equal to:

$$
r=\frac{\sum_{i} x_{i} y_{i}-n \bar{x} \bar{y}}{(n-1) s_{x} s_{y}}
$$

The correlation coefficient is measured on a scale that varies from +1 through 0 to -1 . When one variable increases as the other increases the correlation is positive; when one decreases as the other increases it is negative. Complete absence of linear correlation is represented by 0 .

\section{Interpretation}




$$
\begin{aligned}
& r=0 \quad \text { no linear correlation } \\
& r>0 \quad \text { a positive correlation } \\
& r=1 \quad \text { a perfect positive correlation } \\
& 0<r<1 \quad \text { a partially positive correlation } \\
& r<0 \quad \text { a negative correlation } \\
& r=-1 \quad \text { a perfect negative correlation } \\
& -1<r<0 \quad \text { a partially negative correlation }
\end{aligned}
$$

The strength of the association is regarded as:

$$
\begin{aligned}
& 0<|r|<0.19 \quad \text { very weak } \\
& 0.2<|r|<0.39 \quad \text { weak } \\
& 0.4<|r|<0.59 \text { moderate } \\
& 0.6<|r|<0.79 \quad \text { strong } \\
& 0.8<|r|<1 \quad \text { very strong }
\end{aligned}
$$

\section{Remark}

Excel:

Insert $\rightarrow$ Charts $\mid$ Scatter $\rightarrow$ Layout and then "Linear Trend"

and select "Display R-squared value on chart"

\section{Example 54}

A study was conducted to analyze the relationship between advertising expenditure and sales. The following data were recorded

\begin{tabular}{l|ccccc}
\hline Advertising & 20 & 24 & 30 & 32 & 35 \\
\hline Sales & 310 & 340 & 400 & 420 & 490 \\
\hline
\end{tabular}

Find the best-fit regression equation and compute the correlation coefficient between advertising expenditure and sales.

We can use Excel software:

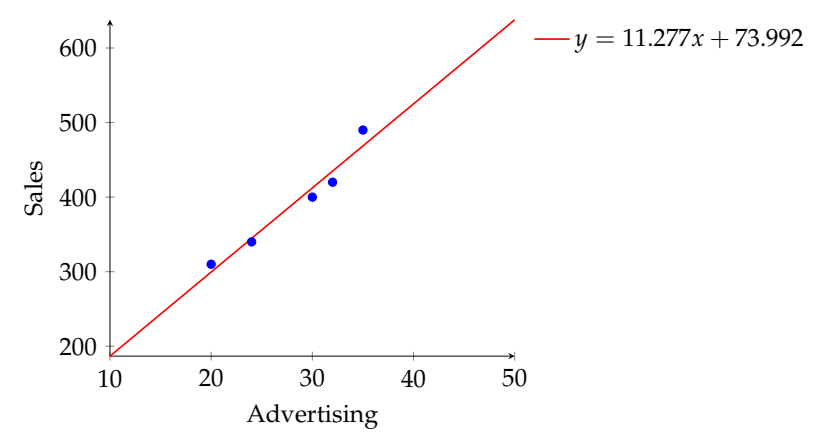


The regression equation is

$$
y=73.992+11.277 x
$$

The coefficient of determination is

$$
R^{2}=0.9518
$$

Finally the coefficient of correlation is

$$
r=\sqrt{R^{2}} \doteq \sqrt{0.9518}=0.9756
$$

The correlation coefficient is 0.9756 , so it indicates a strong positive correlation between advertising expenditure and sales.

A test of significance for a linear relationship between the variables $x$ and $y$ can be performed using the sample correlation coefficient. Let $r$ be the observed correlation coefficient between $x$ and $y$.

We wish to test the hypothesis $H_{0}: \rho=0$ (there is no significant linear relationship between $x$ and $y$ ) against $H_{1}: \rho \neq 0$ (there is a significant linear relationship between $x$ and $y)$.

The test statistics is

$$
t=r \sqrt{\frac{n-2}{1-r^{2}}}
$$

and it follows Student's distribution with $n-2$ degrees of freedom.

\section{Example 55}

For a sample of eight bears, researchers measured the distances around the bears' chests and weighed the bears. The correlation coefficient between the chest size and weight of bears is $r=0.744$ for 8 bears. Using $\alpha=0.05$, determine if there is a positive linear correlation between chest size and weight.

The hypothesis testing problem is $H_{0}: \rho=0$ against $H_{1}: \rho>0$.

The test statistics is

$$
t=0.744 \frac{\sqrt{8-2}}{\sqrt{1-0.744^{2}}}=2.727 .
$$

The critical value of $t$ is $1.943(=\operatorname{T.INV}(0.95 ; 6))$.

Decision - 2 ways:

- $t=2.727>1.943=t_{\alpha} \Rightarrow H_{0}$ is rejected

- $p=1-T . D I S T(2.727 ; 6 ; 1)=0.0172 \Rightarrow p$-value is less than the chosen significance level then we can reject the null hypothesis 
There is sufficient evidence to conclude that there is a significant positive linear relationship between chest size and weight of bears.

\section{Exercise 56}

a) Using $\alpha=0.01$, determine if there is a positive linear correlation between the alcohol content and the number of calories in 12-ounce beer. How strong is this relationship?

\begin{tabular}{l|cccccccc}
\hline Alcohol content (\%) & 4.7 & 6.7 & 8.1 & 4.2 & 5.1 & 5.0 & 5.0 & 4.7 \\
\hline Calories & 163 & 215 & 222 & 104 & 162 & 158 & 155 & 158 \\
\hline
\end{tabular}

b) Following is the data about the demand and price of a commodity for 8 periods. It was expected to estimate a linear regression for demand and price of a commodity. Test whether there is a significant negative relationship between price and demand of a product.

\begin{tabular}{l|cccccccc}
\hline Demand & 16 & 20 & 18 & 21 & 13 & 15 & 17 & 22 \\
\hline Price & 10 & 8 & 12 & 6 & 13 & 9 & 11 & 7 \\
\hline
\end{tabular}


Name: Workbook for Statistics

Department, Institute: Faculty of Mechanical Engineering, Department of Mathematics and Descriptive Geometry

Authors: Petra Schreiberová, Marcela Rabasová

Place, year of publishing: Ostrava, 2021, 1st Edition

Number of Pages: 66

Published: VSB - Technical University of Ostrava

ISBN 978-80-248-4490-9

DOI $10.31490 / 9788024844909$ 